\title{
The patterning behaviour and accumulation of spherical particles in a vibrated non-isothermal liquid
}

\author{
Marcello Lappa \\ Telespazio,Via Gianturco 31 - Napoli, 80046 - Italy, marlappa@unina.it
}

\begin{abstract}
:
A completely new phenomenon of particle accumulation in vibrated non-isothermal monodisperse suspensions of solid spheres (in a liquid) is analyzed. For the first time evidence is provided for this case that even in situations in which particle-particle hydrodynamic interactions are negligible (dilute systems), intriguing non-linear effects can lead to the irreversible formation of well-defined particulate structures over "long" temporal scales, i.e. times much larger than the period of the applied vibrations. The long-range translational ordering is produced by the delicate interplay between convective effects (of thermovibrational nature) and the (inertial) response of each isolated particle to the time-periodic acceleration. A new family of particle attractors in the physical space is identified with the topological dimension being essentially a function of the "symmetry properties" of the considered vibrated system and related geometrical constraints.
\end{abstract}

Keywords: Two-phase dispersive flows, buoyant vibro-convective transport, particle clustering phenomena

\section{Introduction}

Complex fluids are not only of fundamental interest but they are also of practical importance with applications in a variety of areas (mechanical, chemical, petrochemical, nuclear, energy, materials, ocean, mineral and electronics and micro-electronics engineering, information technology, space technology, micro- and nano-technologies, biomedical and life sciences). Such fluids are binary mixtures that have a coexistence between two phases: liquid-liquid (emulsions), liquid-gas (foams), solid-gas (granular), and solid-liquid (suspensions). They are known to exhibit unusual responses to applied force fields due to the geometrical constraints that the phase coexistence imposes and/or effects being produced by the different densities of the component phases.

The resulting properties of the multiphase mixture, in general, depend on several fundamental factors which play a role at the microscopic (particle-size) scale: collisions, hydrodynamic interactions, viscous effects (drag) and inertia. At the same time, the macroscopic appearance can be also linked to more complex issues such as chaotic dynamics, clustering on multiple length scales, and the interplay between these opposite tendencies. Studying these systems, therefore, can be seen as a matter of understanding how ensemble properties arise from the cooperative behaviour 
of sub-parts or different mechanisms (detailed structures, nonlinearities and interdependencies on finer scales).

This field continues to burgeon and bring surprises to this day.

A well-documented phenomenon that can affect a suspension is inertial clustering, i.e. concentration of particles into narrow bands due to their inertia. Historically, the development of this subject took a peculiar path mostly depending on the declared intention of investigators to compare results coming from theoretical models with experiments. Along these lines, several authors confirmed that owing to the finite mass and size of particles their motion tends to deviate appreciably from that of ideal tracers (no mass, vanishing size), which would simply follow the local velocity of the background flow; among such attempts, in particular, it is worth mentioning Balkovsky et al. ${ }^{1}$, who clearly indicated that because of finite particles' inertia, the velocity field of a set of spatially distributed particles will violate the incompressibility constraint even if the fluid flow is incompressible. Today this simple principle is regarded as the necessary theoretical prerequisite allowing solid particles (in association with regions of high shear present in the fluid and a more or less pronounced degree of turbulence of the considered velocity field) to demix and form ordered accumulation structures in specific regions of the physical space ${ }^{1-13}$.

A parallel line of research also exists in which "mechanically forced" particle ordering phenomena have been considered. In such cases, rather than being the mere outcome of spontaneous inertial effects (depending on the nature and properties of the fluid flow), attractive or repulsive events and related patterning behaviour occur essentially as a result of the hydrodynamic interactions among different particles. Such interactions are expressly promoted by the application of mechanical stimuli ("shaking" of the considered system or a body force varying sinusoidally in time).

The identification of the fundamental principles driving such phenomena dates back to the works of Tabakova and Zapruanov ${ }^{14}$ and Langbein ${ }^{15}$. Initial experimental studies are due to Petit and Noetinger ${ }^{16}$, who clearly identified shear-induced crystallization phenomena driven by alternating shear stress in macroscopic suspensions (observations were reported on structures formed by glass spheres in silicon oils and highly viscoelastic fluids, mixtures of polyisobutylenes). Another interesting mean effect of such a spontaneous organisation is the formation of horizontal thin clouds during the sedimentation of granular matter in a liquid subjected to intensive vertical vibration $\left(\right.$ Evesque $^{17}$ ). Moreover, the emergence of vibrational patterns can strongly modify the sedimentation process. Tirumkudulu et al. ${ }^{18}$ extended such studies to suspensions of monodisperse neutrally buoyant spherical particles, thereby demonstrating that (solid vs liquid) density difference is not a necessary pre-requisite. The suspension was indeed observed to separate itself into alternating regions of high and low particle concentration along the length of the tube. Similar results were presented by Wunenburger et al. ${ }^{19}$ and $\mathrm{Zhu}$ and $\mathrm{Ma}^{20}$, who reported on striped domain structures either parallel or perpendicular to the oscillatory direction depending on the considered oscillatory frequency $\omega$ and the amplitude $\gamma$ of the vibrational acceleration. Additional interesting findings on the fascinating mechanisms that may promote the self-assembly of ordered structures in an isothermal liquid (as a consequence of both attractive and repulsive interactions between 
particles) were presented by Voth et al. ${ }^{21}$. The resulting patterns included hexagonally ordered micro-crystallites, time-periodic structures, and chaotic fluctuating patterns with complex dynamics. The related mechanisms have been placed in a precise theoretical context over recent years, see, e.g., the excellent works by Thomson et al. ${ }^{22}$, Ivanova et al. ${ }^{23}$, Kozlov et al. ${ }^{24}$, Hassan et al. ${ }^{25}$, Hassan and Kawaji ${ }^{26}$, Saadatmand and Kawaji ${ }^{27}$. The major outcome of such theoretical efforts has been the derivation of precise mathematical expressions relating the interaction forces to the physical properties of the considered solid-liquid system over a wide spectrum of conditions (viscous or inviscid fluids, low or high frequencies, dilute or concentrated suspensions, etc.). In such a context, it is also worth mentioning the very interesting line of inquiry (where dissipation dominates and inertia is ignored, i.e. Stokesian dynamics) originating from the experiments performed by Pine et al. $^{28}$, who investigated the asymptotic behaviour of solid-liquid systems governed by time reversible equations of motion (essentially collections of neutrally buoyant spheres suspended in a viscous fluid and subjected to a periodic shear). Remarkably, these authors identified a phase transition separating reversible from irreversible dynamics as the imposed strain amplitude or the volume fraction of solid mass were increased (the reader being also referred to the later excellent works ${ }^{29-30}$ ). Most recently there has been an upsurge of interest in all these dynamics owing to the discovery of new patterns and intriguing spatiotemporal phenomena (Pacheco-Martinez et al. ${ }^{31}$ ).

This relatively synthetic review of the literature indicates that the general pattern-formation problem in solid-liquid systems is rich, and several issues are embedded in it. We have seen how two main categories of solid particle attractors have been identified and investigated till date, one coming under the general heading of "inertial phenomena" (requiring solid/liquid density difference, finiteness of particle sizes and masses and some mean background flow transporting otherwise still particles, inter-particle hydrodynamic interactions being not necessary) and the other intimately related to the existence of mechanically excited inter-particle forces (requiring neither solid/liquid density difference nor a "background flow").

The related lines of research are not mutually exclusive, nor are they truly progressive. These contrasts indicate that these problems are cousins rather than twins, and it is very likely that such an intriguing mix of similarities as well as differences will keep investigators engaged for years. In such a context, in the present work we present a new (heretofore unseen) category of vibrationinduced phenomena which seem to escape a possible simple definition or classification in the frame of the two abovementioned main lines of research (still retaining, however, some affinities with the typical features and salient ingredients pertaining to both categories).

More specifically, we consider situations in which the particles tend to acquire a recognizable level of self-organization (i.e. a precise morphology and/or topology in space and/or lines of evolution in time) under the influence of vibrations in very dilute systems (for which therefore hydrodynamic interactions are negligible). The considered configurations, however, are non isothermal, which opens the door to possible effects of thermovibrational nature. Another peculiarity of the considered problem is the absence of a mean (background) flow (which, in general, is essential to promote inertial aggregation). To our knowledge, no theoretical prediction has been proposed concerning these conditions, nor related results have been discussed in the literature. A goal of this study will 
be to achieve sufficient knowledge of the particle association kinetics (so as to be able to control and predict the localization of the fluid particles) and the properties of related "attractors" in the physical space.

\section{Mathematical model}

\section{A. Vibrations}

We are interested in the action of alternating flows on suspensions through nonlinear effects. The specificity of the considered alternating flows is that their linear effects have zero-time averaged value. Hence, only nonlinear effects are expected to be significant even if they are small compared to the instantaneous linear effects. Such alternating flows are produced by a displacement of the considered geometrical system along a given direction ( $\underline{\hat{n}}$ is the related unit vector) varying in time as $\underline{s}^{l a b}(t)=b \sin (\omega \mathrm{t}) \underline{\hat{n}}$ where $\mathrm{b}$ is the displacement amplitude and $\omega=2 \pi f$ is the related angular frequency $^{32-34}$. This is equivalent to imposing a velocity of the considered geometry in the fixed (laboratory) reference system $\underline{V}^{l a b}(t)=V_{\omega} \cos (\omega \mathrm{t}) \underline{\hat{n}}$ (where $V_{\omega}=\mathrm{b} \omega$ ); this, in turn, implies an instantaneous acceleration $\underline{g}(t)=g_{\omega} \sin (\omega \mathrm{t}) \underline{\hat{n}}$ where $g_{\omega}=\mathrm{b} \omega^{2}$. In other words, vibrating a system with frequency $f$ and displacement amplitude $b$ corresponds to a sinusoidal gravity modulation with the same frequency and acceleration amplitude $b \omega^{2}$ and vice versa (accordingly, hereafter the terms "gravity modulation", "periodic acceleration", "system vibration" and g-jitter will be used as synonyms). Such inertial perturbations are known to act on "inhomogeineities" present in the considered fluid system ${ }^{35}$. These inhomogeineities can be due (see, e.g., Ref. ${ }^{11}$ ) to the granular structure of matter itself, as an assembly of hard spheres, or to density inhomogeineities, as those induced in a simple fluid by temperature gradients (which lead to the so-called thermovibrational flow ${ }^{36-39}$ ). The related principles and governing equations are illustrated in the following sections.

\section{B. The Geometry}

The approach is to concentrate on idealized models that incorporate some presumed key aspects. For simplicity, we consider the cubic cavity with characteristic size L shown in Fig.1, delimited by solid walls (one at $\mathrm{z}=-0.5$ cooled, the other at $\mathrm{z}=0.5$ heated, the origin of the coordinate system being located at the cavity center, adiabatic conditions on the remaining walls). The value of the Prandtl number is $\mathrm{Pr}=8\left(\mathrm{NaNO}_{3}\right)$. Moreover, no steady residual gravity is present. The periodic accelerations are contained in the xy plane and form an angle $\phi$ with the $x$ axis. In particular, given the system geometrical symmetries (a replacement of the $\mathrm{x}$ variable with $\mathrm{y}$ would lead to a configuration perfectly equivalent to the initial one), we limit ourselves to considering the range $0 \leq \phi \leq \pi / 4$. 


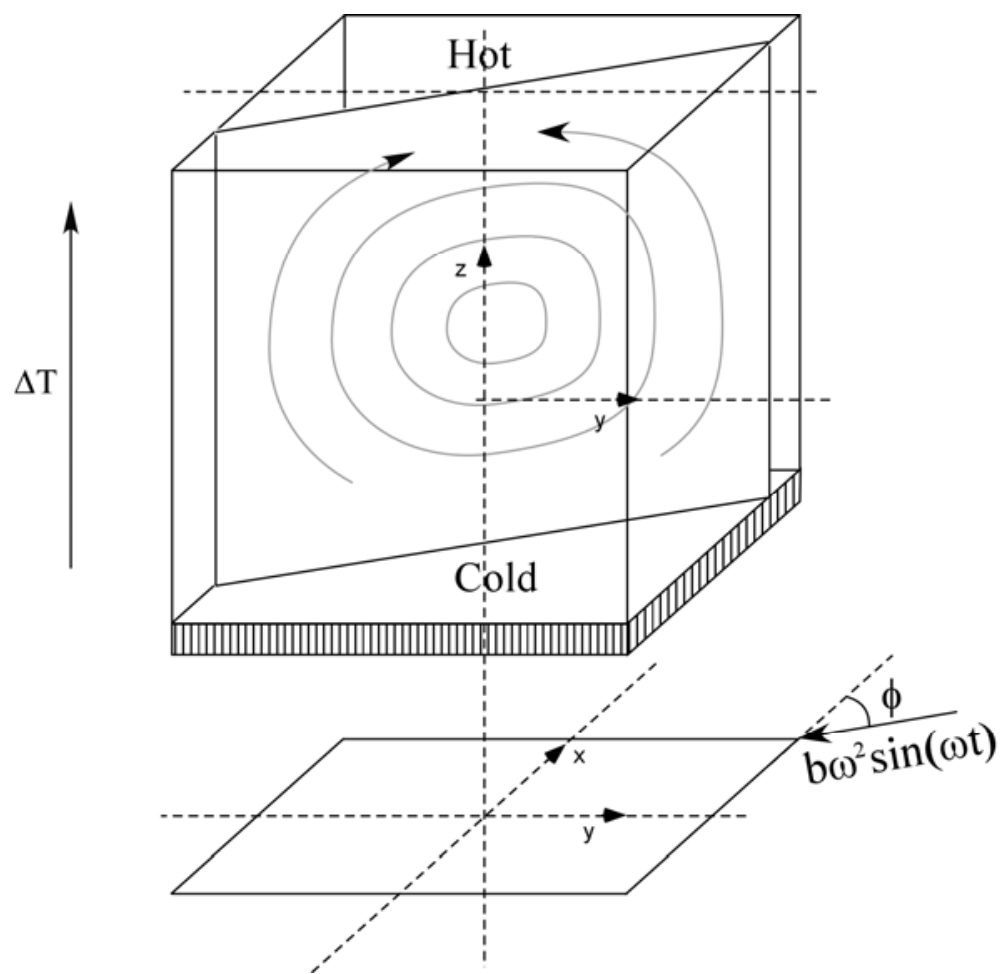

Figure 1: Cubic cavity with characteristic size L shown, delimited by solid walls (one at $\mathrm{z}=-0.5$ cooled, the other at $\mathrm{z}=0.5$ heated, adiabatic conditions on the remaining sidewalls). The vibrations are contained in the xy plane and form an angle $\phi$ with the $\mathrm{x}$ axis $(0 \leq \phi \leq \pi / 4)$.

\section{Nondimensional balance equations for the liquid phase}

Variables are non-dimensionalized using conventional scalings: the co-ordinates by the system extension (L), the velocity components by the energy diffusion velocity $V_{\alpha}=\alpha / L$ where $\alpha$ is the fluid thermal diffusivity, time and pressure by $\mathrm{L}^{2} / \alpha$ and $\rho \alpha^{2} / \mathrm{L}^{2}$, respectively, where $\rho$ is the fluid density. The temperature is measured with respect to its initial value $\overline{\mathrm{T}}_{\mathrm{m}}$ and is made nondimensional as $\mathrm{T}=\left(\overline{\mathrm{T}}-\overline{\mathrm{T}}_{\mathrm{m}}\right) / \Delta \mathrm{T}$ where $\Delta \mathrm{T}$ is the imposed temperature gradient.

In such a framework and using the Boussinesq approximation to account for the variations of fluid density in the body force resulting from the vibration-induced acceleration (as discussed in Sect. 2.1), and keeping all other fluid properties constant otherwise, the continuity and momentum equations simply read:

$\underline{\nabla} \cdot \underline{V}=0$

$\frac{\partial \underline{V}}{\partial t}=-\underline{\nabla} p-\underline{\nabla} \cdot[\underline{V} \underline{V}]+\operatorname{Pr} \nabla^{2} \underline{V}+\operatorname{Pr} \frac{b \omega^{2} \beta_{T} \Delta T L^{3}}{v \alpha} T \sin \left(\frac{L^{2} \omega}{\alpha} t\right) \underline{\hat{n}}$

where $\frac{b \omega^{2} \beta_{T} \Delta T L^{3}}{v \alpha}=R a_{\omega}$ can be regarded as a variant of the classical Rayleigh number (see, e.g., Refs. $^{40-41}$ ) with the steady acceleration being replaced by the amplitude of the considered monochromatic periodic acceleration ( $\beta_{\mathrm{T}}$ is the thermal expansion coefficient). 
In this form the degrees of freedom of the problem are reduced to four independent nondimensional parameters only: the well-known Prandtl number $(\mathrm{Pr}=v / \alpha$ where $v$ is the fluid kinematic viscosity), the nondimensional frequency $(\varpi)$, the nondimensional acceleration amplitude $(\gamma)$ and buoyancy factor $(\beta)$ defined as:

$\varpi=\frac{\omega L^{2}}{\alpha}$

$\gamma=\frac{b \omega^{2} L^{3}}{\alpha^{2}}$

$\beta=\left(\beta_{T} \Delta T\right)$

Accordingly, the momentum equation can be cast in compact form as:

$\frac{\partial \underline{V}}{\partial t}=-\underline{\nabla} p-\underline{\nabla} \cdot[\underline{V} \underline{V}]+\operatorname{Pr} \nabla^{2} \underline{V}+\operatorname{Pr} R a_{\omega} T \sin (\varpi t) \underline{\hat{n}}$

where, obviously $\operatorname{Ra}_{\omega}=\gamma \beta / \operatorname{Pr}$.

The presence of effects of thermovibrational nature requires consideration of the energy balance equation, which in nondimensional form with the considered reference units reads:

$\frac{\partial T}{\partial t}+\underline{\nabla} \cdot[V T]=\nabla^{2} T$

The kinematic conditions to be imposed on the walls simply reflect the well-known no-slip and impermeability properties of solid boundaries $(\underline{V}=0 \rightarrow u=v=w=0$ where $u, v$ and $w$ are the velocity components along $\mathrm{x}, \mathrm{y}$ and $\mathrm{z}$, respectively). We do not report them here explicitly for the sake of brevity (and given their extreme simplicity). For problem closure, such conditions, however, have to be supplemented with those for the energy equation. As anticipated in Sect. 2.2, the lower $(\mathrm{z}=-0.5)$ and upper $(\mathrm{z}=0.5)$ walls of the domain are assumed to be at uniform and constant (nondimensional) temperatures $T_{0}=0$ and $T_{1}=1$ respectively, while the sidewalls are adiabatic, i.e. the $\partial T / \partial \mathrm{n}=0$ condition is imposed there.

\section{The Dispersed Phase}

Obviously, the dispersed phase introduces additional degrees of freedom in the considered problem (particle shape, size and density). Related nondimensional parameters are the ratio of the particle to the fluid density, $\xi$, and the Stokes number, defined a $\mathrm{St}=\tau \nu / \mathrm{L}^{2}$ where $\tau$ is the so-called particle relaxation time $\left(\tau=2 R^{2} / 9 v\right)$ and $R$ is the particle radius (particles are assumed to be perfectly spherical). 
The present problem may be therefore regarded as a typical example of situations involving an interplay between large-scale (thermovibrational flow) and small-scale entities and processes (particles and their motion under the influence of large-scale flow and periodic accelerations), which, in general, require a multiscale philosophy. Following the same approach undertaken in an earlier work ${ }^{11}$, here particles are treated as isolated, microscopic quantities compared with field variables, but their motion is tracked by using an appropriate equation (taking into account inertia, the added mass effect and viscous drag forces, see, e.g. ${ }^{42-43}$ ):

$\frac{d \underline{V}_{\text {partcl }}}{d t}=\frac{1}{\xi+1 / 2}\left[-\frac{\operatorname{Pr}}{S t}\left(\underline{V}_{\text {partcl }}-\underline{V}\right)+\frac{3}{2} \frac{d \underline{V}}{d t}+\frac{3}{2}(\underline{V} \cdot \underline{\nabla} \underline{V})\right]+\frac{\xi-1}{\xi+1 / 2} \gamma \sin (\varpi t) \underline{\hat{n}}$

where $\underline{V}_{\text {part }}\left(u_{\text {part }}, v_{\text {part }}, w_{\text {part }}\right)$ and $\underline{V}$ are the particle and fluid velocity, respectively (the Maxey-Riley equation). Such equation does not account for a retroactive influence of particles on fluid motion (it is based on a one-way coupling assumption, i.e. particle motion cannot alter the liquid flow). Moreover, particles are assumed to be independent of each other (this is permissible if the concentration of the dispersed phase in the flow is small, i.e. if the distance of a particle from others is much larger than their diameter, see again Ref. ${ }^{11}$ and references therein). Despite such approximations, however, tracers moving close to the solid boundaries require a special treatment (given the no-slip properties of such surfaces acting as sinks of momentum and, therefore, as a potential particle-entrapping loci). Following earlier studies, in particular, we assume particles to interact in a non-elastic fashion with walls (in practical numerical implementation, this means a particle can approach the solid boundary until at a distance not smaller than its radius is achieved; then it is allowed to slide along the boundary until the wall-normal velocity component becomes directed inwards).

The underlying assumptions on which this approach is based are, therefore, particles with solid/fluid density ratio $\xi \neq 1$, a perfectly spherical shape, non-elastic particle-wall interaction and a small value $(<<1)$ of the particle to fluid system characteristic size ratio $\mathrm{R} / \mathrm{L}$ (which implies a particle response time much less than the characteristic time scales of the considered flow, this condition being a necessary prerequisite for the validity of the Maxey-Riley equation ${ }^{11}$ ).

\section{E. Solution Method and Validation Study}

Balance equations (1-6) have been solved numerically by a time-explicit finite-difference method (primitive-variable method) based on a three-dimensional rectangular mesh and a staggered collocation of fluid-dynamic variables. Forward differences in time and central-differencing schemes in space (second order accurate) have been used to discretize the energy and momentum governing equations. The related solution strategy is not discussed here, the interested reader being referred to various books and articles in the literature (e.g., Ref. ${ }^{44}$ ) for an exhaustive treatment (for the implementation of this method on parallel machines, the reader may consider, e.g., Ref. ${ }^{45}$ ). The part concerning the computation of particle motion with equation (6) was validated in earlier studies of the present author ${ }^{11}$. 


\section{F. Scheme Order and Error Analysis}

Equation (6) has been dynamically integrated together with equation (1,3-5) (i.e. with the same time integration step) over a mesh $42 \times 42 \times 42$. Given the small value of the nondimensional time integration step $\Delta \mathrm{t}$ required for the numerical stability of the Navier-Stokes algorithm $\left(\mathrm{O}\left(10^{-}\right.\right.$ $\left.\left.{ }^{6}\right)<\Delta \mathrm{t}<\mathrm{O}\left(10^{-5}\right)\right)$, one is not forced to use high-order schemes for the integration of eq. (6). Even with a first-order scheme, in fact, the error produced at each step (the so-called local truncation error) is $\mathrm{O}\left(\Delta \mathrm{t}^{2}\right)$, which accumulated over the number of iterations $\mathrm{N}_{\text {iter }}$ required for the formation of particle accumulation (typically $\mathrm{N}_{\mathrm{iter}} \cong \mathrm{O}\left(10^{6}\right)$ for the conditions considered here), gives a global truncation error $\left(\right.$ Atkinson $\left.^{46}\right) \mathrm{N}_{\text {iter }} \Delta \mathrm{t}^{2}=\mathrm{O}\left(10^{-5}\right)$.

\section{Results and discussion}

The main objective of the present study is to understand how vibrations can eventually induce particle ordering mechanisms in a dilute non-isothermal solid-liquid system (a dispersion of spherical particles in liquid $\mathrm{NaNO}_{3}$ ).

Microgravity conditions are considered. On Earth, particles suspended in a fluid are largely confined to the depth where buoyancy and gravity forces balance out, so studies of their selforganization, in general, are limited to two dimensions, whereas in real zero gravity, as on the International Space Station, suspended particles can assemble in three dimensions (PachecoMartinez et al. ${ }^{31}$ ). This is a subject of importance, not only for discovering new phenomena but also for evaluating the effect of the unavoidable vibrations, which may, in some cases considerably perturb fluid and material science experiments (see the excellent studies conducted by Beysens ${ }^{47}$; see also Ref. $\left.^{11}\right)$.

The properties of the considered solid-liquid system are summarized in Table I.

TABLE I: Liquid-solid system properties

Fluid $\left(\mathrm{NaNO}_{3}\right)$ density $\rho, \mathrm{kg} \mathrm{m}^{-3}$

Melting temperature $\mathrm{T}_{\mathrm{m}}, \mathrm{K}$

Kinematic viscosity $v, \mathrm{~m}^{2} \mathrm{~s}^{-1}$

Kinematic viscosity temperature coefficient $v_{\mathrm{T}}, \mathrm{m}^{2} \mathrm{~s}^{-1} \mathrm{~K}^{-1}$

Thermal diffusivity $\alpha, \mathrm{m}^{2} \mathrm{~s}^{-1}$

Thermal expansion coefficient $\beta_{\mathrm{T}}, \mathrm{K}^{-1}$

Particle/liquid density ratio $\xi$

$\mathrm{L}, \mathrm{m}$

$\Delta \mathrm{T}, \mathrm{K}$
1904

581

$1.27 \times 10^{-6}$

$-3.6 \times 10^{-9}$

$1.58 \times 10^{-7}$

$1.25 \times 10^{-3}$

1.85

$2 \times 10^{-2}$

67

The very small value of the kinematic viscosity temperature coefficient $v_{\mathrm{T}}$ (Nunes et al. ${ }^{48}$ ) allows to consider negligible the variations of kinematic viscosity with temperature ${ }^{7}$ up to values of $\Delta \mathrm{T} \cong 70$ $\mathrm{K}$ (for which the percentage variation experienced by $v$ is only $10 \%$, in agreement with the constant 
viscosity model defined in Sect. IIC). Assuming for instance as size of the cubic cell $\mathrm{L}=2 \times 10^{-2} \mathrm{~m}$ and a fixed temperature difference $\Delta \mathrm{T} \cong 67 \mathrm{~K}$ and varying the amplitude and angular frequency of the acceleration disturbance in the ranges $4.6 \times 10^{-4} \leq \mathrm{g}_{\omega} \leq 4.6 \times 10^{-3} \mathrm{~ms}^{-2}$ and $0.04 \leq \omega \leq 0.4 \mathrm{~Hz}$ (corresponding in nondimensional form to $1.5 \times 10^{5} \leq \gamma \leq 1.5 \times 10^{6}$ and $10^{2} \leq \varpi \leq 10^{3}$, respectively), the characteristic nondimensional number $\mathrm{Ra}_{\omega}$ (see eq. (4)) spans the range $1.58 \times 10^{3} \leq \mathrm{Ra}_{\omega} \leq 1.58 \times 10^{4}$. By keeping the amplitude of the system velocity in the fixed reference frame $V_{\omega}$ constant (this being equivalent to considering the ratio $\gamma / \varpi=$ const), however, all these conditions correspond to a given value of the so-called Gershuni number:

$$
G s=\frac{\left(b \omega \beta_{T} \Delta T L\right)^{2}}{2 v \alpha}=\frac{\left(\beta_{T} \Delta T L\right)^{2}}{2 v \alpha}\left(\frac{g_{\omega}}{\omega}\right)^{2}=\frac{1}{2 \operatorname{Pr}}\left(\frac{\beta \gamma}{\varpi}\right)^{2}
$$

Such a parameter (which, in general, accounts for the intensity of time-averaged convection, see, e.g., Ref. ${ }^{11}$ ), is fixed to Gs $=10^{3}$, so as to guarantee that time-averaged convective effects of thermovibrational nature are very small with respect to the oscillatory component of fluid velocity. This also guarantees a negligible departure of the time-averaged temperature field from the purely linear and diffusive conditions (in the absence of observational information to properly constrain the model parameters, our goal is to demonstrate that even the current "minimal" model can produce the desired effect).

The density of the particles is also assumed to be fixed ( $\xi=1.85)$. A degree of freedom, however, is introduced in the problem by changing the characteristic particle size via the value of the Stokes number St or its related parameter $\eta=(\xi-1) \operatorname{St} / \operatorname{Pr}$ (accounting for the combined effect of particle inertia and drag, hereafter simply referred to as the "inertia parameter", Lappa ${ }^{10}$ ).

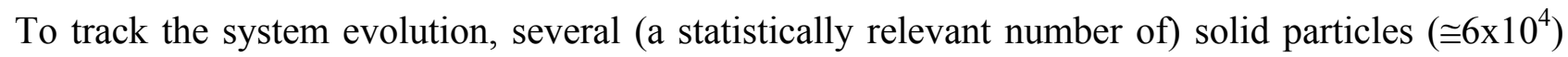
are initially seeded uniformly into the computational domain. Given the one-way-coupling approach discussed in Sect. 2.4, such a relatively high number of particles (apparently violating the initial assumption of dilute solid-liquid system) is used for visualization purposes only. It should be recalled in fact that in the present framework the outcomes of the computations in terms of patterning behaviours and related evolution times do not depend on the amount of used solid tracers; the particle attractors revealed by the numerical simulations are an intrinsic property of the flow: even a single particle would be attracted by them (the number of particles effectively used having meaning only in the level of accuracy in a statistical sense with which the model can represent the threedimensional structure of the attractor). As particle dynamics, however, do depend (via eq. (6)) on the kinematic conditions established in the surrounding liquid, the initial velocity of all tracers is set equal to zero (to reflect the initial conditions assumed for the liquid phase, i.e. $\underline{V}=\underline{V}_{\text {part }}=0$ at $\mathrm{t}=0)$.

\section{A. Tubular Structures}

We first concentrate on the more instructive case of vibrations aligned with the $\mathrm{x}$ axis $(\phi=0$, Fig. 1). 
According to the numerical results, if the parameter $\eta$ is smaller than a given limit $\left(\eta \cong 10^{-4}\right)$, welldefined structures are formed in the physical space. As shown in Fig. 2, in particular, the individual particles organize themselves into what appears to be a twodimensional pattern (the dependence on the y coordinate is relatively weak). More precisely, particles order along 3D surfaces whose intersection with xz planes gives a couple of closed (reducible) curves in the considered plane (see, e.g., Fig. 5 f for $y=0)$.

As the characteristic size and shape of these two curves do not change much in different xz planes, this gives the illusion of two main tubes parallel to the y axis (Fig. 2).

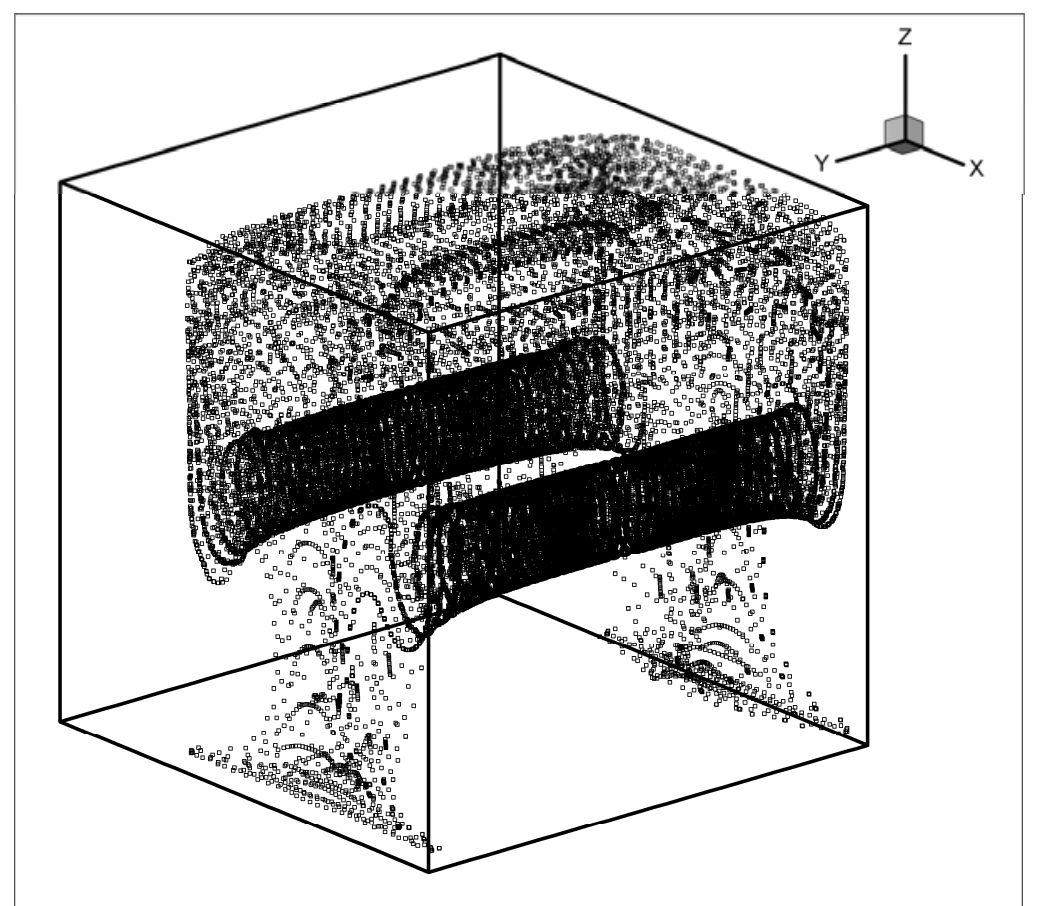

Figure 2: Particle aggregates (3D view at $\mathrm{t}=7 \times 10^{-1}$ ) for $\omega=1 \times 10^{3}, \gamma=1.5 \times 10^{6}, \mathrm{Ra}_{\omega}=1.58 \times 10^{4}, \xi=1.85$, and $\eta=1 \times 10^{-4}$ (vibrations along the $\mathrm{x}$ axis, $\phi=0$ ).

Remarkably, although if the motion of a single particle was simulated the final position taken by the particle on the attractor would depend on its initial position, the asymptotic morphology and topology of the steady structures collectively formed by the statistically relevant number of particles considered here do not depend on their initial distribution; only the transient does and, in such a context, particularly interesting is the interplay between the transport of solid particles by largescale flow, on the one hand, and convective phenomena in fluid phase induced by periodic accelerations, on the other hand. Before embarking in such a description, let us recall that, in a nonisothermal system, vibrations perpendicular an imposed temperature gradient are known to generate time-dependent vorticity ${ }^{31,33-35}$. The vorticity is produced essentially by the interplay between the temperature distribution and the instantaneous acceleration caused by vibrations (see Sect. IIA). More precisely, such acceleration and the imposed temperature gradient will alternatively cooperate to produce a clockwise or anticlockwise convective cell in the $\mathrm{xz}$ plane over a cycle of the modulation as shown in Fig. 3. 
Phys. Fluids, Vol. 26, No 9, 093301, (2014)
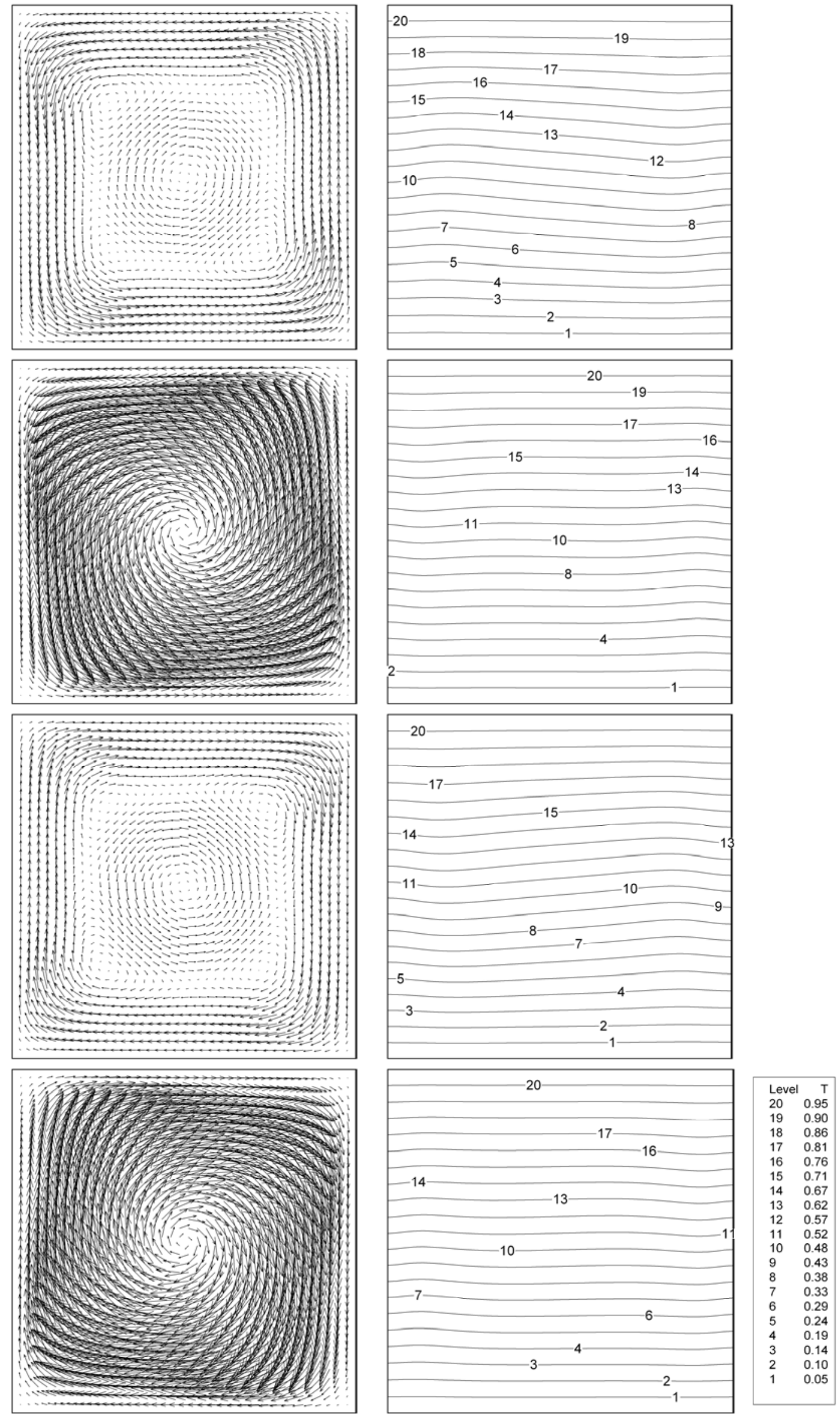

Figure 3: Snapshots of velocity and temperature fields (plane $\mathrm{xz}$, section $\mathrm{y}=0$ ) evenly distributed during one period of oscillation $\left(\varpi=1 \times 10^{3}, \mathrm{Ra}_{\omega}=1.58 \times 10^{4}\right.$, cold side on the bottom $(\mathrm{z}=-0.5)$, hot side on the top $(\mathrm{z}=0.5)$, adiabatic sidewalls, vibrations along the $\mathrm{x}$ axis, $\left.\phi=0, V_{\max } \simeq 25\right)$. 
In that part of the cycle where the acceleration is directed along the positive $\mathrm{x}$ axis direction, the resulting convective cell will be anticlockwise oriented, vice versa (clockwise oriented roll when $\mathrm{g}_{\omega}(\mathrm{t})$ changes its sign).

It is also worth recalling that, in general, the thermofluid-dynamic distortions induced by a periodic zero-mean value acceleration are made up by an average contribution plus a time-periodic component $^{33}$ and that, as outlined at the beginning of Sect. 3, for a not too high value of the Gershuni number the time-averaged departure from purely thermally diffusive and time-averaged purely quiescent conditions (a linear distribution of temperature along the $\mathrm{z}$ axis and a zero average velocity) will be relatively small with respect to the oscillatory component of such a departure.
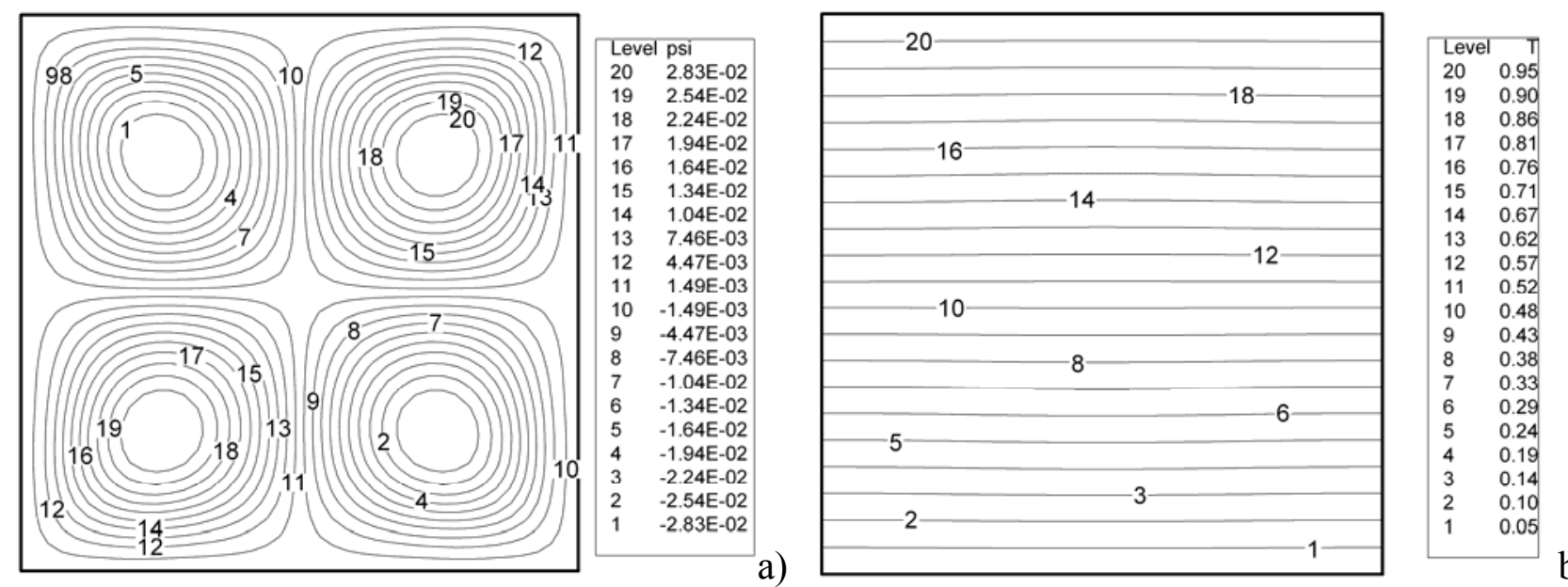

b)

Figure 4: Time-averaged velocity (streamlines) and temperature field (plane $x z$, section $y=0$ ) in the case of vibrations acting along the $\mathrm{x}$ axis and $\varpi=1 \times 10^{3}, \mathrm{Ra}_{\omega}=1.58 \times 10^{4}$, corresponding to $\mathrm{Gs}=10^{3}$ (cold side on the bottom $(\mathrm{z}=-0.5)$, hot side on the top $(\mathrm{z}=0.5)$, adiabatic sidewalls, $\left.V_{\mathrm{max}} \cong 2 \times 10^{-1}\right)$.

Along these lines, comparison of the convective pattern and particle aggregates provides evidence that the key factor for the formation of the pattern is not the existence of an average flow in time (the so-called and well-known quadrupolar field with a four-vortex structure shown in Fig. 4a, which is symmetrical by reflection with respect to both $\mathrm{x}=0$ and $\mathrm{z}=0$ planes, see, e.g., Mialdun et al. $^{49}$ ), but rather the mechanism produced by the instantaneous (oscillating in time) flow (Fig. 3 ) in combination with vibration-induced particle time-displacements (whose nonlinear effects on particles accumulate over time leading to the observed ordered particulate structures).

This is clearly shown in Fig. 5, which provides the spatio-temporal stages of evolution of the considered system from the initial condition with particles uniformly distributed in the fluid up to the emergence of the final pattern with closed curves.

At the beginning (Fig. 5a) edge effects tend to cluster particles close to the walls into two separate lines extended along the direction perpendicular to the imposed vibrations. 
Fig. 5 shows that when a group of particles moving under the effect of the vibration-induced periodic force comes in proximity to one of the physical boundaries, particles tend all to be accumulated along the boundary direction. When the periodic force changes its sign and particles are moved in the opposite direction, the above process results in the appearance of particle-dense lines separating the region containing particles from the pure liquid (particle-depleted region). When such particle-dense lines, subsequently undergo the convective transport due to the largescale thermovibrational flow, they are stretched and deformed producing curved branches bounding the particle region.

In particular, the mechanism of pattern formation consists basically of a progressive translation away from the walls of the abovementioned sharp lines and ensuing folding of their "lower" part (the part located in the cold region of the plane) towards the interior (Figs. 5b). Such a folding is due essentially to the instantaneous motion of fluid shown in Fig. 3, which is alternatively accelerated from the hot to the cold side (due to buoyancy) along the right or left wall (depending on the instantaneous direction of acceleration) and then released in the cold (lower) region of the square section.

This process leads in the initial stages of evolution to the formation of an upside down amphora-like shape as seen in the xz plane (the distribution of particles results in a shape that bulges out above $\mathrm{z}=0$ and necks in below it.). As time increases, the progressive folding of the lines marking the boundary of the region with particles produces two rounded patches "hanging" from the main particle region towards the clear fluid outside (Figs. 5c-d). Such patches are finally transformed in two closed circuits due to the collapse of the related necks (Fig. 5e). The recognizable shapes formed by particles then tend to be stable in time. Indeed, the subsequent evolution consists essentially of a rhythmic left-right displacement of the overall pattern and related characteristic independent loops, which means that the system response to the imposed vibration is synchronous (or harmonic) (particles and velocity fields oscillating at the same frequency of the imposed sinusoidal forcing). 
Phys. Fluids, Vol. 26, No 9, 093301, (2014)

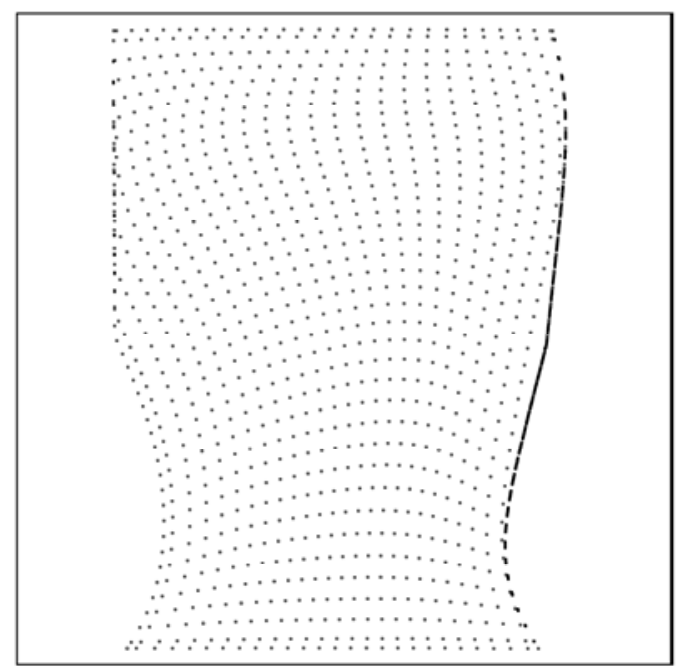

a)

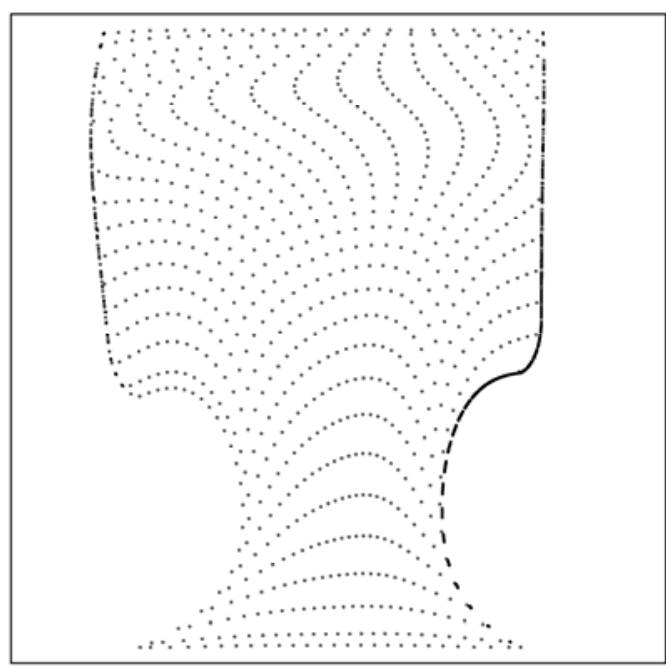

b)
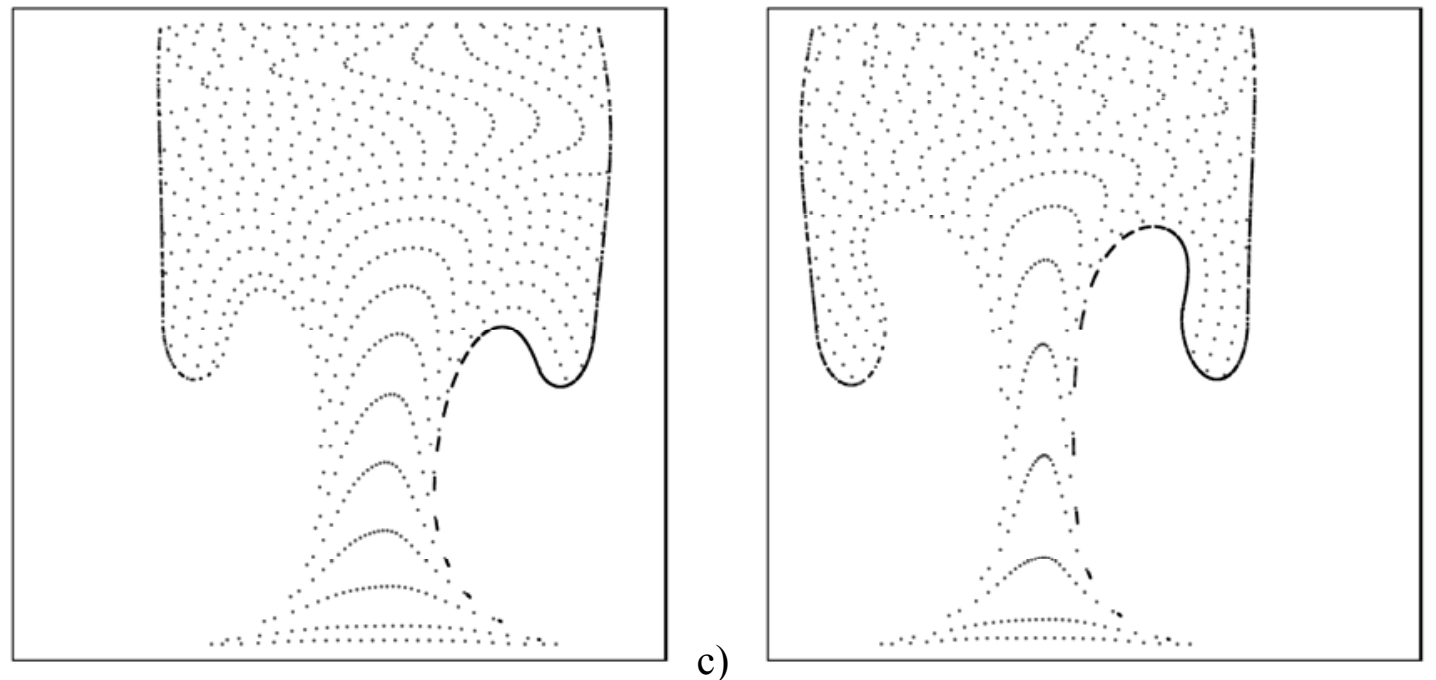

d)
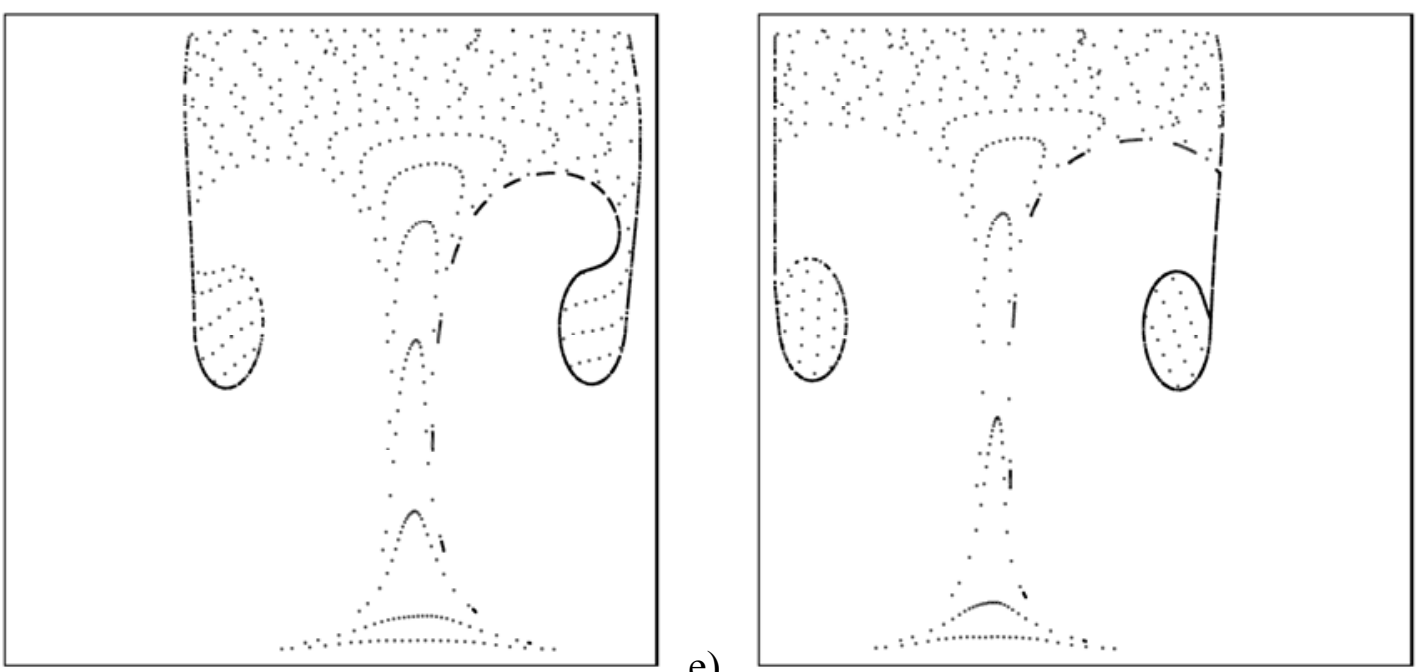

f)

Figure 5: Particle aggregates (snapshots in the section $\mathrm{y}=0)$ as a function of time $\left(\varpi=1 \times 10^{3}\right.$, $\gamma=1.5 \times 10^{6}, \mathrm{Ra}_{\omega}=1.58 \times 10^{4}, \xi=1.85, \eta=1 \times 10^{-4}$ ) (vibrations aligned with the $\mathrm{x}$ axis, $\left.\phi=0\right)$ : a) $\mathrm{t}=2 \times 10^{-2}$, b) $\mathrm{t}=6 \times 10^{-2}$, c) $\mathrm{t}=1 \times 10^{-1}$, d) $\mathrm{t}=1.4 \times 10^{-1}$, e) $\left.\mathrm{t}=1.8 \times 10^{-1}, \mathrm{f}\right) \mathrm{t}=2.2 \times 10^{-1}$. 
In other words, once the two particle circuits are formed, a further increase of time, produces no variation in the morphological and topological properties of the pattern. Under the effect of vibrations the pattern oscillates back and forth along the $\mathrm{x}$ direction apparently as $a$ whole at the vibration frequency as experienced by an observer in the laboratory (fixed) reference frame (compare, e.g., Figs 5e and 5f; although the overall pattern is shifted to the right in Fig. 5e and to the left in Fig. 5f, the characteristic size of the two circuits and their distance remain constant). Since all the particle accumulation structures oscillate in phase with each other and with the same amplitude, the two closed neighbouring lines, maintain a fixed distance in time (although it should be pointed out that, in general, the property of all particle pertaining to the pattern of moving collectively such that an illusion of a unique moving solid item is created only applies to the pattern as such and not to individual particles).

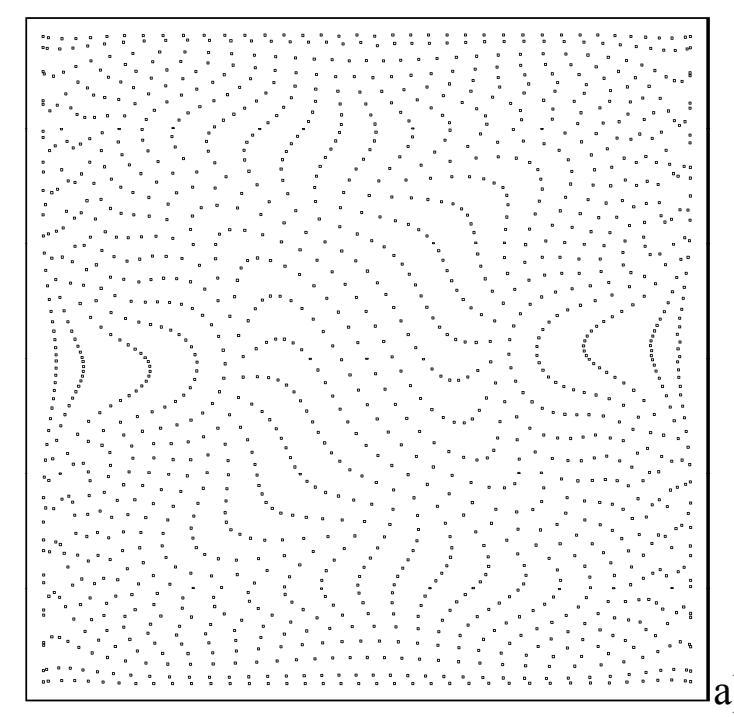

Without particle forces

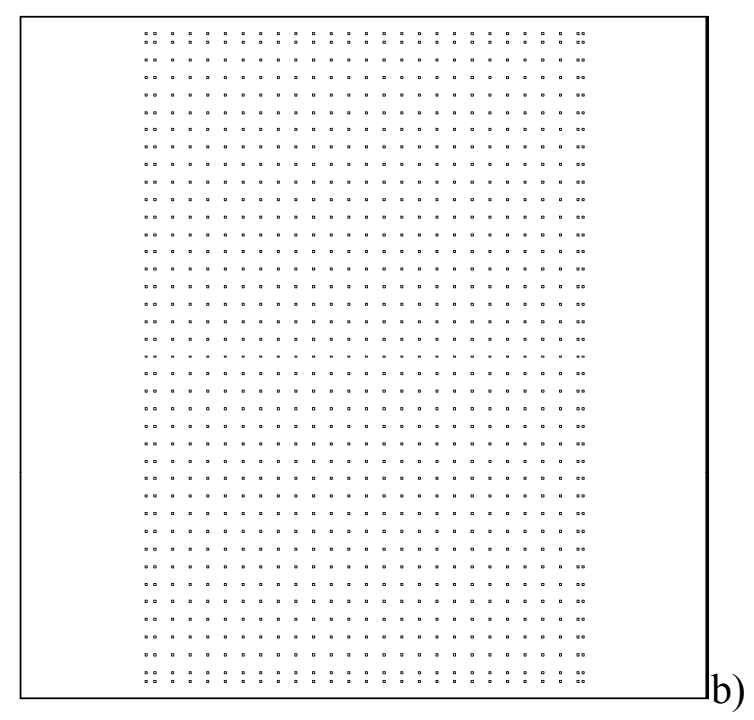

Without vibrational flow

Figure 6: Particle distribution (snapshots in the section $y=0$ for $\varpi=1 \times 10^{3}, \gamma=1.5 \times 10^{6}, \operatorname{Ra}_{\omega}=1.58 \times 10^{4}$, $\left.\xi=1.85, \eta=1 \times 10^{-4}, \mathrm{t}=5 \times 10^{-1}\right)$ : a) without particle forces (liquid with imposed temperature gradient and related oscillatory flow of vibrational nature, but effects of vibrations on particles neglected), b) without vibrational flow (effect of vibrations on particles retained, but fluid in isothermal conditions). This figure demonstrates indirectly that inertial forces and thermovibrational convection support each other cooperatively in the process leading to particle ordering and accumulation.

As a further proof of the synergy between convective effects (of thermovibrational nature) and the response of each isolated particle to the time-periodic acceleration in determining the resulting pattern, Fig. 6 shows that the formation of well-defined structures (in the form of twodimensional surfaces created by the accumulation of particles) is prevented when one of these two effects is not taken into account. 


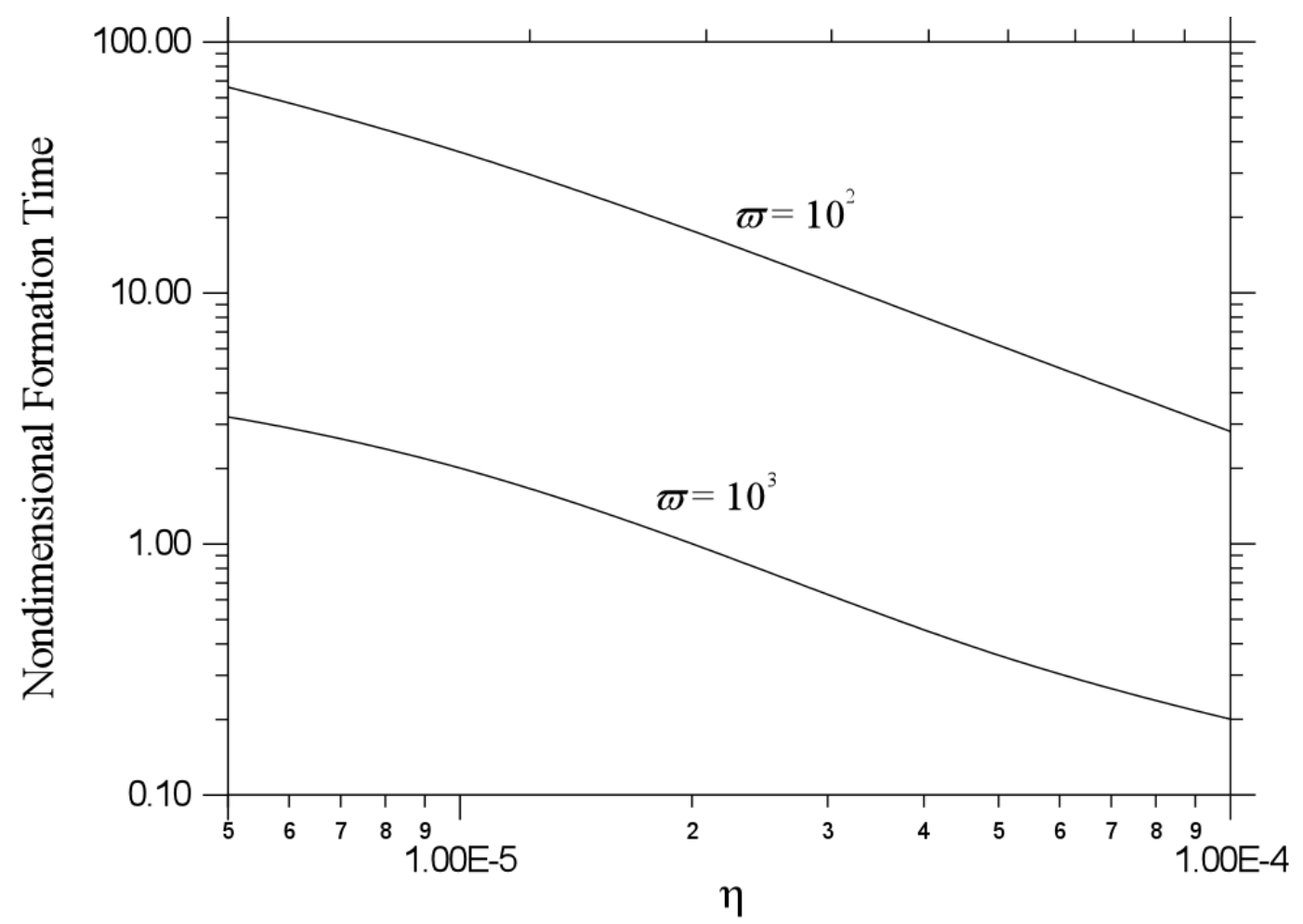

Figure 7: Formation time versus particle inertia $\left(\gamma / \varpi=1.5 \times 10^{3}, \mathrm{Gs}=10^{3}, \xi=1.85\right)$.

Further insights into this phenomenon are provided by an analysis of its dependence on the typical problem parameters (vibration frequency and particle characteristic size). The strong dependence of the formation time on the parameter $\eta$ (Fig. 7), indeed, provides evidence for its "inertial" nature (which, however, is not a sufficient condition, as witnessed by the results in Fig. 6b).

The translation of the curve (Fig. 7) towards higher values of the time for decreasing $\varpi$ clearly indicates that thermovibrational effects also play a crucial role. When the shaking is applied, the transient leading to the final pattern and its duration depend strongly on both vibration frequency and particle size. As shown in Fig. 7, the formation time scales as $(\eta \varpi)^{-1}$.

The inertia parameter, however, has also a strong impact on the characteristic extension of the two circuits in the xz plane (which behaves as a decreasing function of $\eta$, see Figs. 8 and 9). By having an influence on the "efficiency" by which the convective flow is able to transport them (or, in other words, on the ability with which particles are able to "follow" this oscillatory flow), the mass and size of particles will definitely have an important role also in the "folding process" of the particledense lines (discussed before), which finally determines the extension of the closed circuits. More precisely, because these inertial particles interact with the fluid through viscous Stokes drag and, therefore, the motion of particles at a given value of the Stokes number $\mathrm{St}_{2}$ typically lags behind that that would be typical of tracer particles moving with $\mathrm{St}_{1}<\mathrm{St}_{2}$, an increase in $\mathrm{St}(\eta)$ must be expected to decrease the extension of the final particle loops. 
Phys. Fluids, Vol. 26, No 9, 093301, (2014)
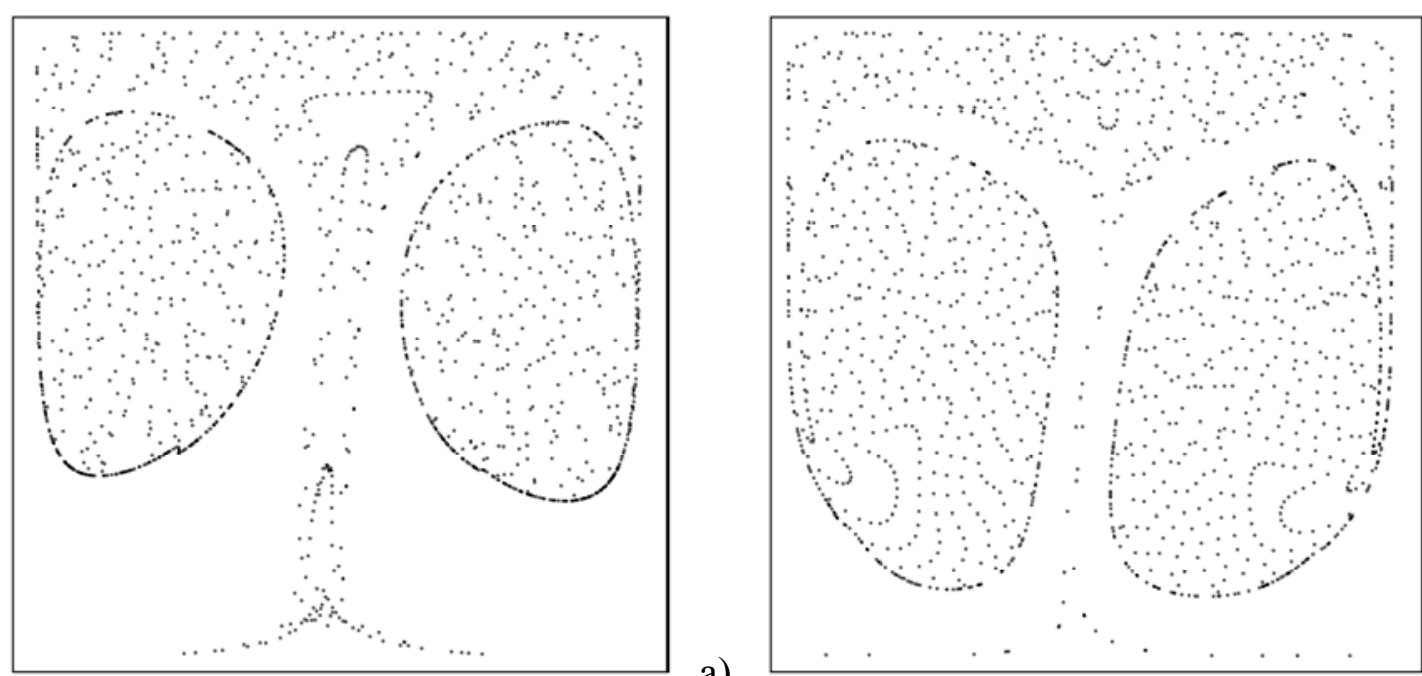

d)
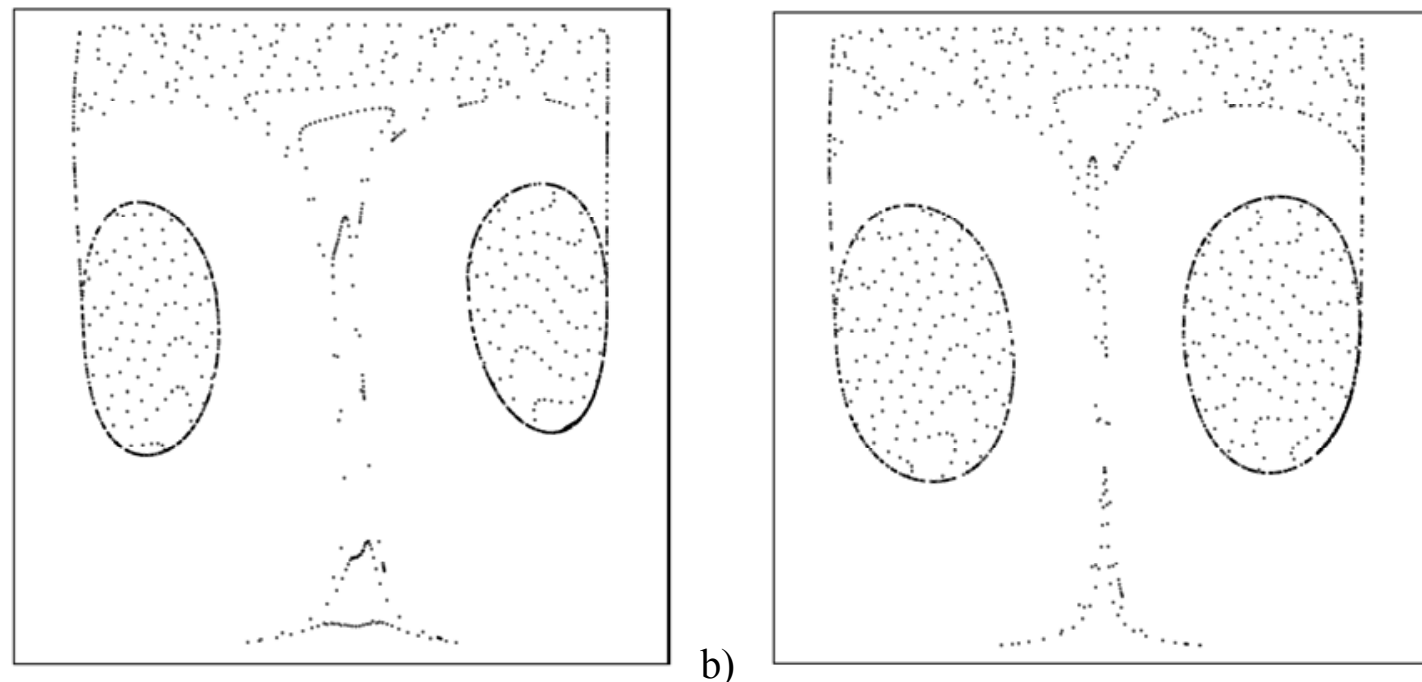

e)
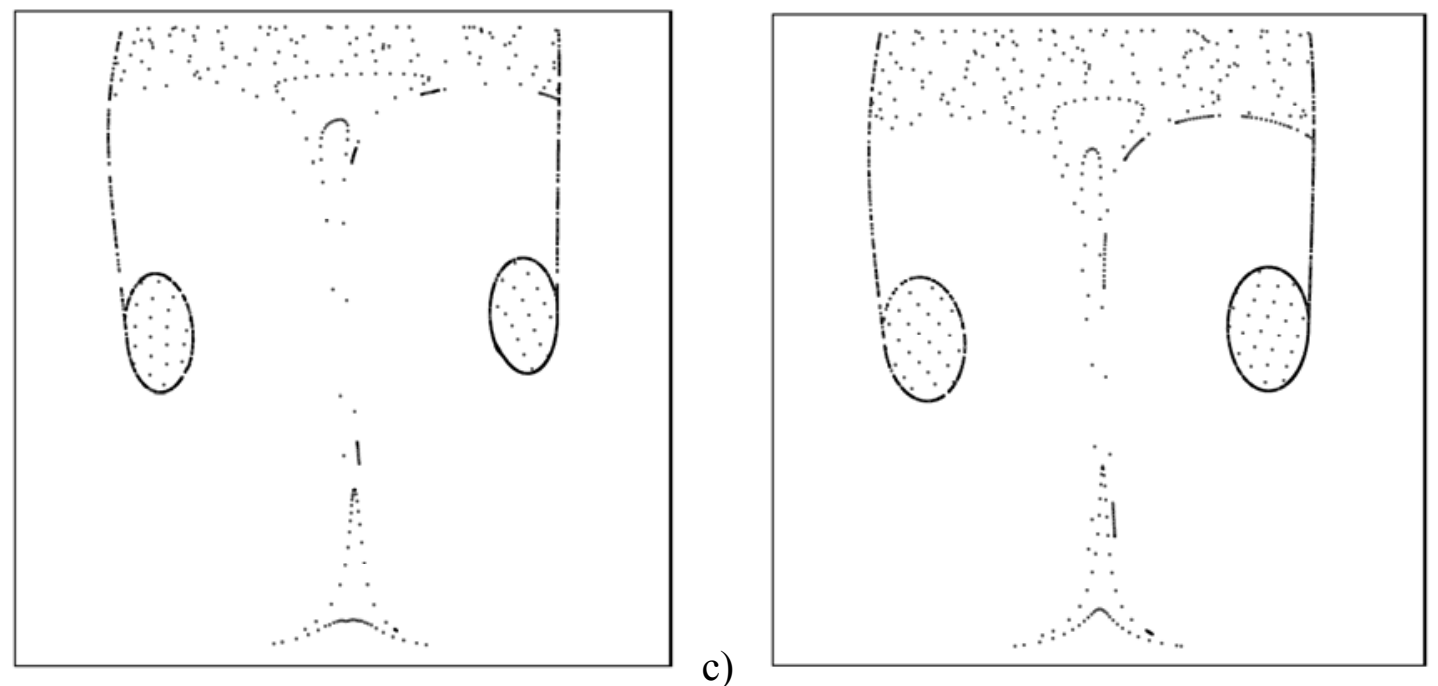

f)

Figure 8: Particle aggregates (snapshots in the section $y=0)$ as a function of $\eta\left(\gamma / \varpi=1.5 \times 10^{3}\right.$, $\mathrm{Gs}=10^{3}, \xi=1.85$ ) (vibrations aligned with the $\mathrm{x}$ axis, $\phi=0$ ): a) $\omega^{2}=1 \times 10^{3}, \eta=1 \times 10^{-5}$, b) $\varpi=1 \times 10^{3}$, $\eta=5 \times 10^{-5}$, c) $\varpi=1 \times 10^{3}, \eta=1 \times 10^{-4}$, d) $\varpi=1 \times 10^{2}, \eta=1 \times 10^{-5}$, e) $\varpi=1 \times 10^{2}, \eta=5 \times 10^{-5}$, f) $\varpi=1 \times 10^{2}$, $\eta=1 \times 10^{-4}$. 
Phys. Fluids, Vol. 26, No 9, 093301, (2014)

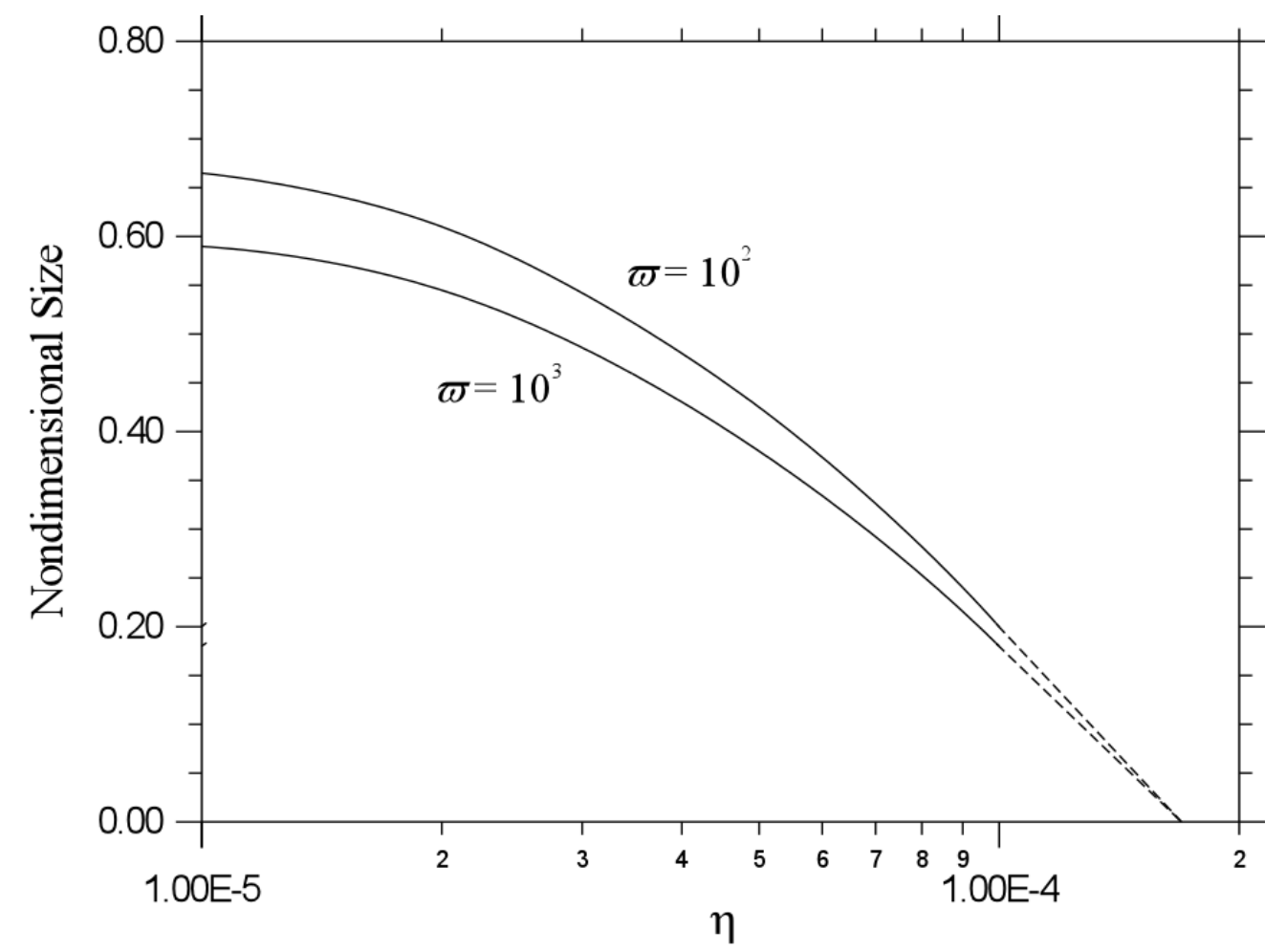

Figure 9: Characteristic size of particle aggregates versus inertia parameter $\left(\gamma / \varpi=1.5 \times 10^{3}, \mathrm{Gs}=10^{3}\right.$, $\xi=1.85)$.

Remarkably, linear extrapolation of the characteristic size (shown in Fig. 9) to zero gives $\eta \cong 1.6 \times 10^{-4}$, which is in agreement with the absence of recognizable extended particle structures in the numerical simulations performed for $\eta=2 \times 10^{-4}$ (see Fig. 10).

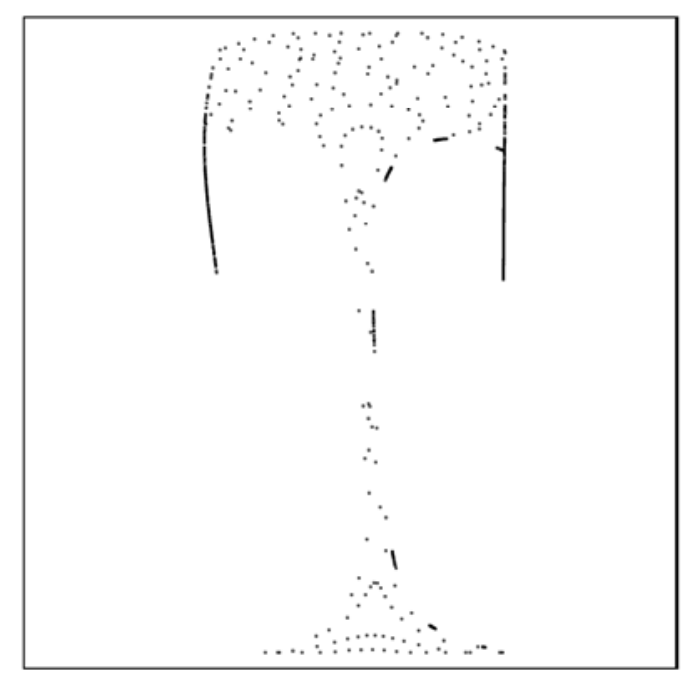

Figure 10: Particle aggregates (snapshots in the section $y=0$ ) for $\gamma=1.5 \times 10^{6}, \varpi=1 \times 10^{3}$, $\operatorname{Ra}_{\omega}=1.58 \times 10^{4}, \xi=1.85, \eta=2 \times 10^{-4}$. 


\section{B. Compact Structures}

In this section the other potential degree of freedom of the considered configuration is explored, i.e. the role played by the possible orientation (inclination) of vibrations in the xy plane $(\phi \neq 0)$ in connection with the intrinsic reflection symmetries of the considered geometrical configuration (these being the two mirror images, and the two diagonal flips shown in Fig. 11).

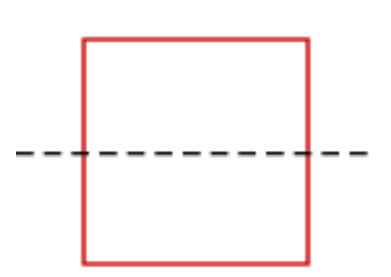

M1 reflection

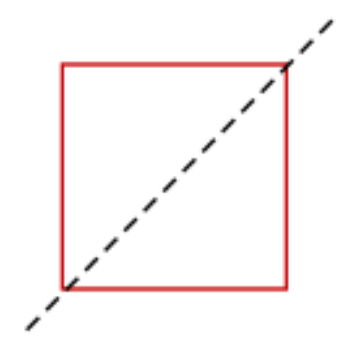

M2 reflection

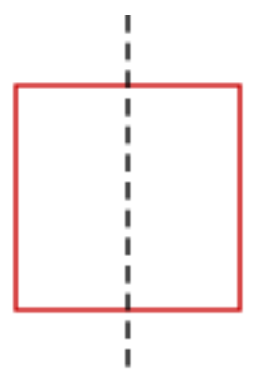

M3 reflection

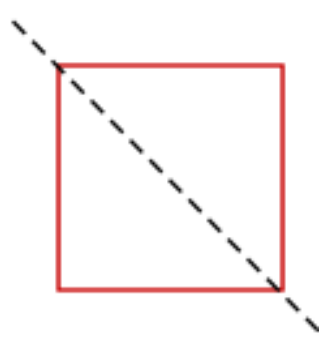

M4 reflection

Figure 11: The symmetry group of a square (reflection symmetries).

Since (as the reader will immediately realize on the basis of the geometrical arguments shown in Fig. 11) the condition $\phi=\pi / 2$ would lead to the same results already seen for $\phi=0$ (the only difference being the orientation of the tubular structures along $\mathrm{x}$ - instead of $\mathrm{y}$-axis, given the replacement of the M3 with the M1 reflection property), in particular, we concentrate initially on the intermediate condition $\phi=\pi / 4$ (vibrations along the direction $y=x$ plane, i.e. the $\mathrm{M} 2$ reflection condition).

Most interestingly, the numerical results reveal that such a simple change in the considered reflection property leads to dramatic variations in the morphological and topological details of the emerging pattern. Indeed, the aggregates formed by the accumulation of particles in the physical space assume the typical properties of "compact" surfaces (a kind of irregular ovoids under a certain perspective, see Fig. 12). 


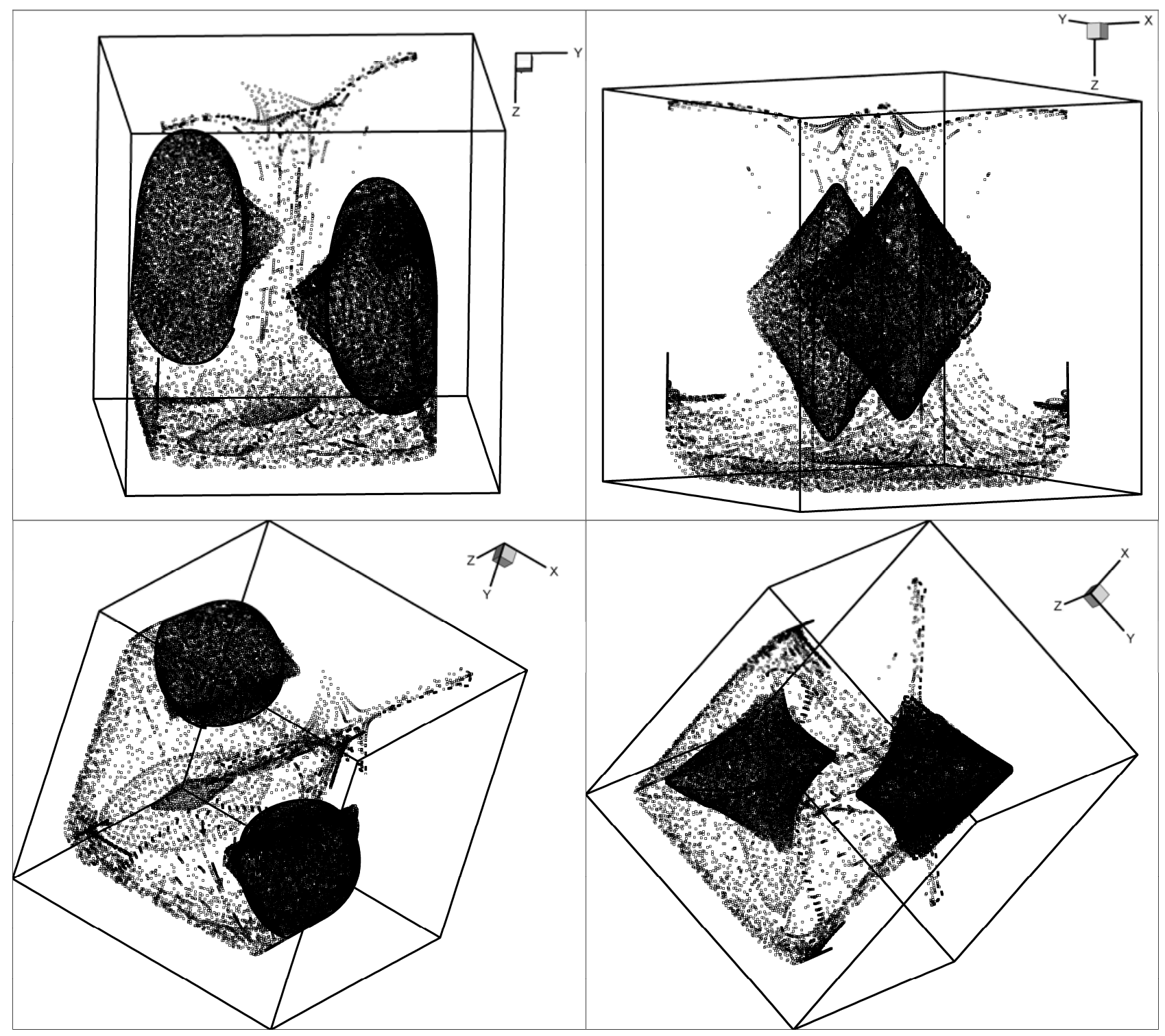

Figure 12: Particle aggregates (different views at $\left.\mathrm{t}=7 \times 10^{-1}\right)\left(\varpi=1 \times 10^{3}, \gamma=1.5 \times 10^{6}, \mathrm{Ra}_{\omega}=1.58 \times 10^{4}\right.$, $\xi=1.85, \eta=1 \times 10^{-4}$, vibrations along the $\mathrm{y}=\mathrm{x}$ direction, $\left.\phi=\pi / 4\right)$.

An oval (from Latin ovum, "egg") is a closed curve in a plane loosely resembling the outline of an egg. The ovoid is the 3D version of an oval. Although this term is not very specific, here we use it simply to indicate the compact appearance of particle aggregates. In fact, one may get different ideas of the resulting shapes according to the point of view used to look at them (as an example, the right frames of Fig. 12 would give the illusion of structures resembling a couple of polyhedrons rather than the couple of rounded geometrical items visible in the left frames).

With the above arguments we have attempted to assemble a simple, intuitive and reasonably selfcontained discussion of the main geometrical properties of the observed sets of particles. Leaving aside considerations on the effective shape taken by the aggregates, however, the major outcome of such simulations is the demonstration of the increased ability of walls in constraining the resulting accumulation structures when the properties of the vibrated geometrical system change from the M1 or M3 reflection symmetry to the M2 or M4 one. 
Such increased "ability" should be seen as the consequence of a change in the structure and orientation of the convective flow induced by the instantaneous vibration-induced acceleration. It, in fact, is no longer contained in xz planes (as it was in Fig. 3) and develops velocity components along the $\mathrm{y}$ direction (comparable in intensity to those along the $\mathrm{x}$ direction), which make the general concepts discussed in Sect 3.1 and illustrated in Fig. 5 applicable to the yz plane as well. The "folding action" exerted by fluid being released in the cold region on the boundary separating the region of particle accumulation from that of particle depletion becomes effective in both $\mathrm{xz}$ and yz planes. This argument provides a relatively simple justification for the observed change in the shape of particle aggregates from tubular surfaces to compact structures.
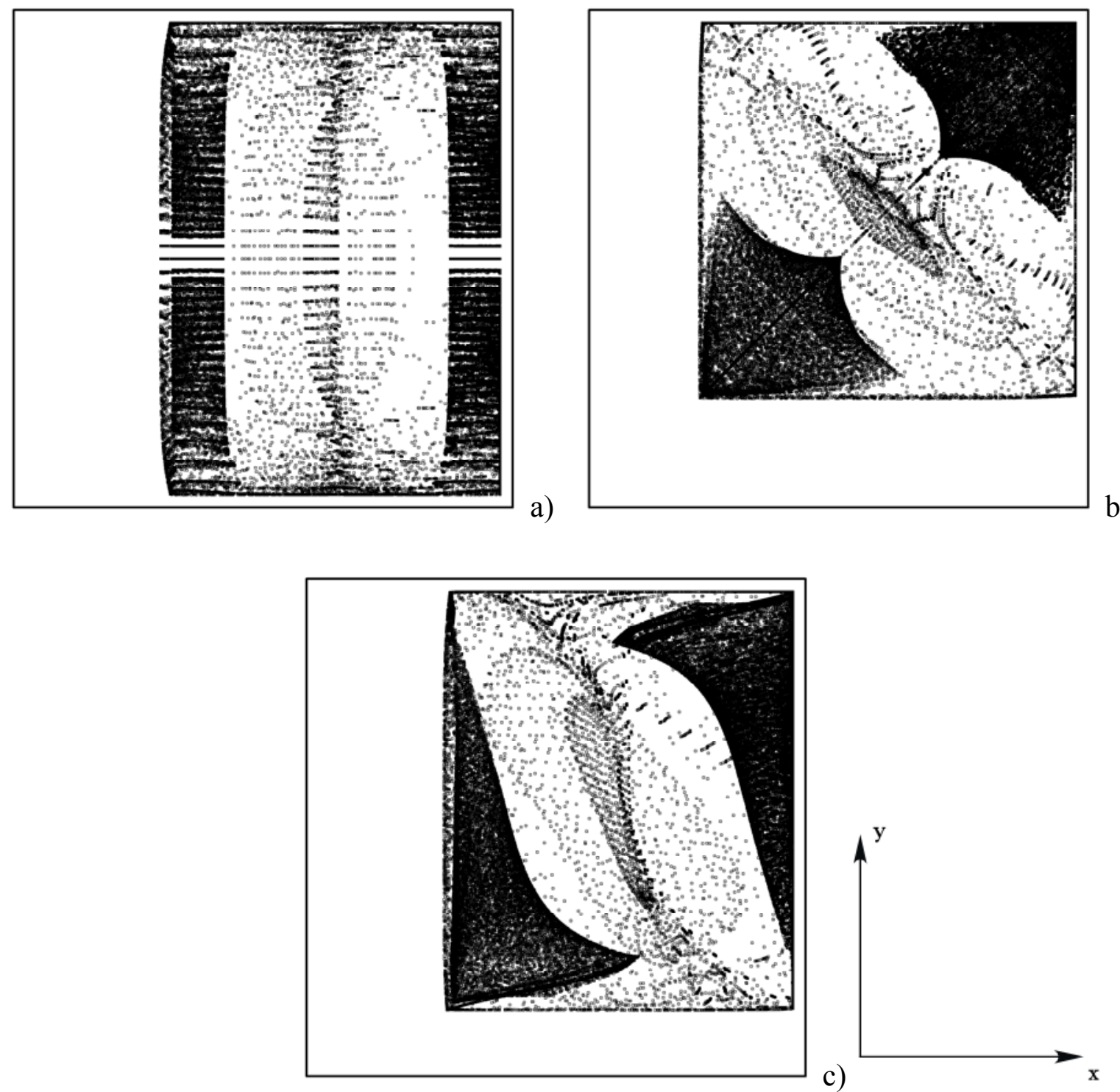

Figure 13: Particle aggregates (top view) $\left(\varpi=1 \times 10^{3}, \gamma=1.5 \times 10^{6}, \operatorname{Ra}_{\omega}=1.58 \times 10^{4}, \xi=1.85, \eta=1 \times 10^{-4}\right.$, $\mathrm{t}=7 \times 10^{-1}$ ) for different values of the vibrations angle $\phi:$ a) $\phi=0$, b) $\phi=\pi / 4$, c) $\phi=\pi / 8$ (the external square box indicates the boundary of the cubic cell containing the liquid). Particles initially dispersed in the fluid collect into a seemingly rigid structure that apparently moves as a unit under the effect of vibrations. 
Interestingly, as evident in Fig. 13 (which shows a top view of the system) the extension along the $\mathrm{x}$ and $y$ directions of the region affected by the presence of particles (in such a figure the white area between the external system boundary and the internal dark region indicates the zone that has been completely depleted of particles) depends on the angle of imposed vibrations.

For $\phi=0$ a compression of the pattern formed by particles is clearly visible along the $\mathrm{x}$ direction, whereas its extension along the y axis covers almost the entire cavity. For $\phi \neq 0$, some shrinkage of the pattern becomes visible also along $y$ (with compression rate depending on the effective value of $\phi)$ until it achieves the same degree of compression along both the $\mathrm{x}$ and $\mathrm{y}$ axes for $\phi=\pi / 4$.

In all cases, the particle clusters move back and forth along the direction of imposed vibrations as apparently solid items (as already discussed to a certain extent for the particular case $\phi=0$ when commenting on Fig. 5).

It is also worth mentioning that, regardless of whether the reflection symmetry is even (M2 or M4) or odd (M1 or M3), the geometric items formed by the particle accumulation process retain the symmetry property of the vibrated system (as the two particle circuits in Fig. 8 are symmetric with respect to a vertical plane $\mathrm{x}=$ const, the items in Fig. 12 are symmetric with respect to $\mathrm{y}=\mathrm{x}$, see Fig. $13 b)$.

Such a feature, however, is no longer valid when the direction of the imposed vibrations is such that the reflection symmetries shown in Fig. 11 are violated (that is $\phi \neq n \pi / 2, \phi \neq n \pi / 2+\pi / 4$ where $\mathrm{n}=1,2,3,4)$. This is clearly shown in Fig.13c, which provides the top view of particle aggregates for $\phi=\pi / 8$.

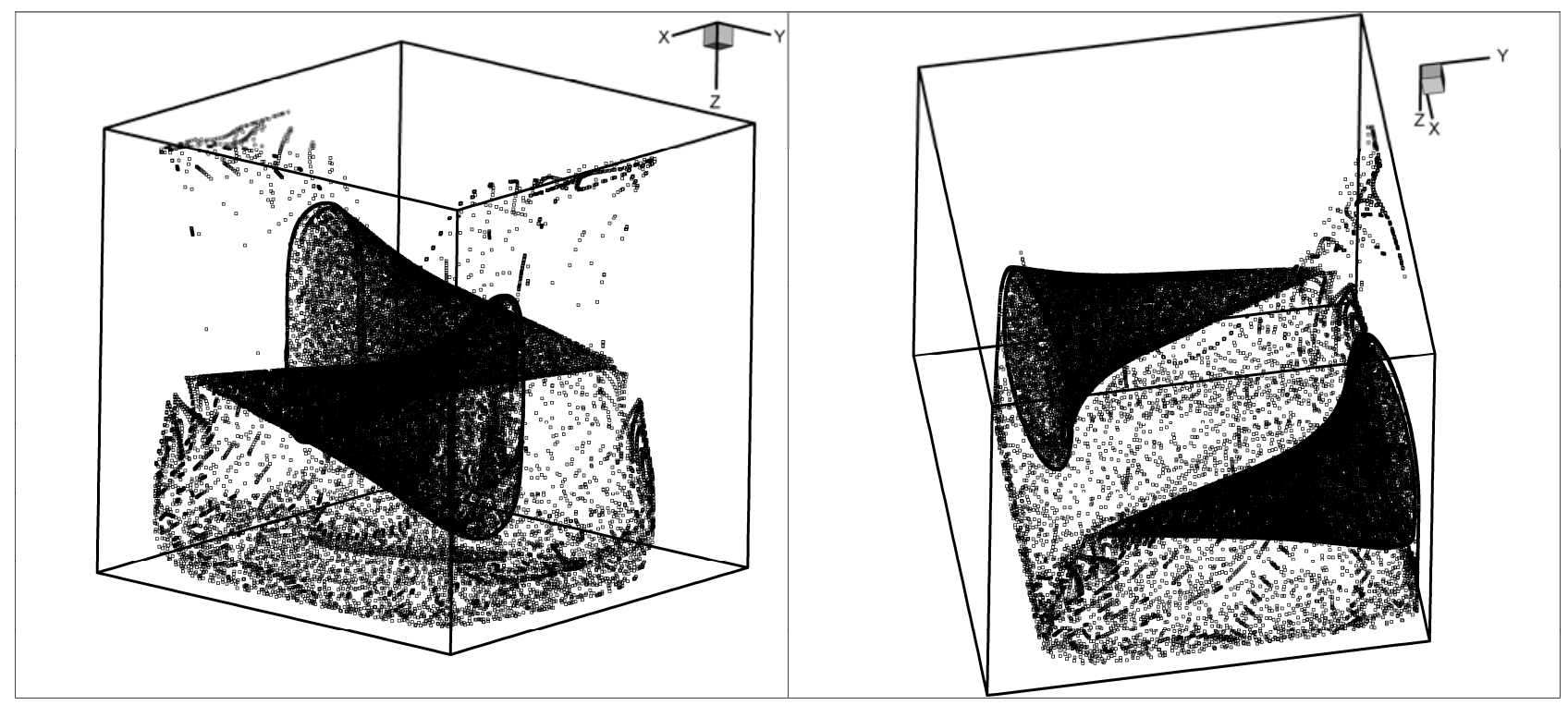

Figure 14: Particle aggregates (two different views at $\left.\mathrm{t}=7 \times 10^{-1}\right)\left(\varpi=1 \times 10^{3}, \gamma=1.5 \times 10^{6}\right.$, $\left.\mathrm{Ra}_{\omega}=1.58 \times 10^{4}, \xi=1.85, \eta=1 \times 10^{-4}, \phi=\pi / 8\right)$. 
Although the two geometric items formed by the particle accumulation process for $\phi=\pi / 8$ may be regarded one as the opposite (upside down) mirror image of the other (and, remarkably, this property has been found to be valid for all cases with $0<\phi<\pi / 4)$, the relatively simple reflection symmetries seen in Figs. 13a and 13b are no longer present. Moreover, interestingly, the loss in fundamental symmetry is accompanied by a significant change in the morphology of the resulting particle surfaces. Both take the shape of a distorted conical surface (the set of points formed by the union of more or less curved lines passing through a fixed point, the apex, and any point of a special closed circuit, the directrix curve, which in the present case, as shown in Fig. 14, is approximately elliptic). Such a kind of conic surfaces may be regarded as the intermediate configuration between the compact ovoids/polyhedrons represented in Fig. 12 for $\phi=\pi / 4$ and the tubular surfaces seen in Fig. 2 for $\phi=0$.

Such findings on the other hand are surprisingly in line with the well-known geometrical property of a cylindrical surface to be potentially interpretable as a limiting case of a conical surface whose apex is moved off to infinity in a particular direction (in projective geometry a cylindrical surface is just a special case of a conical surface). Such a limit case corresponds in the present case to the situation in which vibrations are perfectly perpendicular to the sidewall $(\phi=0)$.

Most notably, such simple arguments may be the hint for a possible affinity between the present dynamics and the geometrical concept of a quadric surface. As we have seen, when the angle $\phi$ increases from 0 to $\pi / 4$, the particle accumulation structure evolves from a cylindrical surface to a compact surface passing through the intermediate stage of a conical surface, which may indicate a possible isomorphism with the general laws of projective geometry (see, e.g., Bennett ${ }^{50}$ ). The basic intuition behind this idea is that as a projective space has more points than the Euclidean space, in a given dimension, and geometric transformations are permitted when the extra points (called "points at infinity") are moved to traditional points (and vice versa), the considered fluid-dynamic system may behave in the same way. Just as the ellipsoid and the elliptic paraboloid, are non-degenerate compact quadric surfaces, while the cone and the cylinder are equivalent to the so-called degenerate form, the particle surfaces revealed by the present study may be the signature of some hidden analytic order (algebraic equation) underlying the formation and existence of such attractors in the physical space.

Given the complexity and level of abstraction required by such an analysis, here we renounce to attempt the identification of such precise laws, which might be the main subject of future studies. Rather, as a final point still to be discussed, we emphasize again that although for all cases considered, the particle structures visible in the ideal reference system oscillating in space with the pattern at the same frequency of imposed vibrations, are essentially stationary (their properties do not change in time), however, as time increases such structures often exhibits local crystalline defects, which consist in defects and clusters of particles that result in line thickening and local pattern compression-dilation. These defects, which arise from interaction of the particle with boundaries, evolve slowly in time (such that, as repeatedly mentioned earlier, the illusion of a solid structure undergoing a synchronous translational motion in space with applied vibrations, in general, is retained). 


\section{Conclusions}

Particle segregation is a counterintuitive process where an initially mixed state of a fluid and granular matter separates into its constituent components. Such phenomena are frequently observed in the presence of mechanical excitation, but there is no unified theory, and even the pertinence of the description in terms of sole inertial (background-flow-dependent) or sole hydrodynamic (particle-particle) effects is still an open question.

The main objective of this work was to understand if/how mechanical excitation (in the form of vibrations or "gjitters") could induce ordering mechanisms in a dilute, but non-isothermal, disperse system (in other words, whether a category of phenomena may exist in mechanically excited systems for which inter-particle hydrodynamic interactions play no role while density difference and thermal convective effects are essential).

As the problem is extremely complex (it is not easy to discern the complex interrelations among the various parameters under one's control that are not independent of one another), much care has been devoted to characterize unambiguously the salient ingredients that can influence the probability of success in inducing phase separation and particle ordering. To exclude the possibility of particle association (or, by contrast, particle dispersion) driven by a mean (time-averaged) background flow, the Gershuni number has been kept constant and very small. To explore system sensitivity to vibrations and particle properties, the nondimensional vibrations frequency and the particle Stokes number have been allowed to change over two or more orders of magnitude.

The main point of our analysis is that, despite the low value of the Stokes number $\left(\mathrm{St} \leq 10^{-3}\right)$, inertia and drag effects, acting in combination with the role exerted by solid walls in limiting particle motion and determining accumulation in specific regions of the physical space, are responsible for the ordering over very long times compared with the time scale of the considered problem (the period of oscillatory vibrations). The associated time scale (pattern formation time) depends on both the Stokes number and nondimensional vibration frequency, ranging from $\mathrm{O}(10)$ of vibration periods for $\mathrm{St} \cong 10^{-3}$ to $\mathrm{O}\left(10^{3}\right)$ for $\mathrm{St} \cong 5 \times 10^{-5}$. An increase in $\varpi$ determines a significant decrease in the formation time (one order of magnitude when $\varpi$ is increase from $10^{2}$ to $10^{3}$ ); a similar effect is produced by an increase in the Stokes number (or its related parameter $\eta$ ).

The above-mentioned dependence on St and the lack of particle aggregates when vibration-induced forces acting on particles are neglected provides a clear indication that inertia (particle size and a mass density different from that of the carrier fluid) does play a role in the considered category of phenomena; the failure in obtaining ordered particle structures when the system is isothermal, however, also demonstrates that time-dependent (oscillatory) flow of thermovibrational nature is an essential ingredient (average-flow being absent or negligible given the very low value of the Gershuni number).

In other words, the spatio-temporal behaviour of the emerging patterns depends on both the dynamic coupling of the particles with the surrounding fluid medium (as quantified by the Stokes 
number) and the properties of the mechanical excitation (frequency, acceleration amplitude). Last, but not least, the relative direction of applied vibrations with respect to the imposed temperature gradient and the axes of symmetry of the considered geometrical configuration also determines the morphology of the surfaces delimiting the particle attracting regions in the physical space, these being closed (a kind of irregular ovoids) or non-compact (tubular or conic surfaces) depending on which of the intrinsic reflection symmetries of the vibrated system are preserved or violated.

Most notably, such simple observations and ensuing theoretical arguments imply that taking only one of the models traditionally used in the literature (the one assuming recognizable particle structures to be formed by inter-particle hydrodynamic interactions, or the other focusing on inertial effects produced by the interaction of particles with a background mean flow) may be omitting important components of the general dynamics of vibrated systems, especially when they are nonisothermal. Along these lines, it also becomes clear that future studies shall be devoted to assess the influence of particle interactions on the phenomena considered here for the specific case of concentrated systems. It is expected that interparticle attractive or repulsive behaviors may perturb in some way the "stability" and morphology of these fascinating attractors (whose mechanism, as repeatedly explained above, envisions a cooperative action exerted on particles by inertial forces and thermovibrational convection, as demonstrated by the fact that the formation of well-defined structures is prevented when one of these two effects is not taken into account).

The understanding of all these effects will give information on the undesired (destabilising) effects produced by structure vibrations on the numerous typical experiments carried out on orbiting platforms using heterogeneous media. The present results, however, also suggest that our model system may provide useful information for the elaboration of a novel and simple way to control the ordering morphology of inclusions in a number of effective materials-processing techniques. Indeed, the combined use of vibrations and related convective effects may help controlling the motion of particles not only in microgravity but also on the ground where this may be used for phase separation of heterogeneous media in typical industrial applications.

\section{References}

[1] E. Balkovsky, G. Falkovich, and A. Fouxon, Intermittent Distribution of Inertial Particles in Turbulent Flows, Phys. Rev. Lett. 86, 2790-2793 (2001).

[2] M.R. Maxey, B.K. Patel, E.J. Chang, L.-P. Wang, Simulations of dispersed turbulent multiphase flow, Fluid Dyn. Res., 20(1-6), 143-156, (1997).

[3] J. Bec, Fractal clustering of inertial particles in random flows, Phys. Fluids, 15, L81-L84, (2003). J. Bec,. Multifractal concentrations of inertial particles in smooth random flows, J. Fluid Mech., 528, 255-277 (2005).

[4] D. Schwabe, A.I. Mizev, M. Udhayasankar and S. Tanaka, Formation of dynamic particle accumulation structures in oscillatory thermocapillary flow in liquid bridges, Phys. Fluids, 19(7), 072102, (2007).

[5] E. W. Saw, R. A. Shaw, S. Ayyalasomayajula, P. Y. Chuang, and A. Gylfason, Inertial Clustering of Particles in High-Reynolds-Number Turbulence, Phys. Rev. Lett., 100, 214501, (2008). D. Di Carlo, J. F. Edd, K. J. Humphry, H.A. Stone, and M. Toner, Particle Segregation and Dynamics in Confined Flows, Phys. Rev. Lett., 102, 094503, (2009).

[6] T. Sapsis and G. Haller, Clustering criterion for inertial particles in two-dimensional time-periodic and three-dimensional steady flows, Chaos, 20, 017515, (2010).

[7] D. Melnikov, D. Pushkin, and V. Shevtsova, Accumulation of particles in time-dependent thermocapillary flow in a liquid bridge. Modeling of experiments, Eur. Phys. J. Special Topics, 192, 29-39 
(2011). D. O. Pushkin, D. E. Melnikov, V. M. Shevtsova, Ordering of Small Particles in One-Dimensional Coherent Structures by Time-Periodic Flows, Phys. Rev. Lett., 106, 234501, (2011).

[8] Lappa M., Rotating Thermal Flows in Natural and Industrial Processes (John Wiley \& Sons, Chichester, England, 2012).

[9] D. E. Melnikov, D. O. Pushkin, and V. M. Shevtsova, Synchronization of finite-size particles by a traveling wave in a cylindrical flow, Phys. Fluids 25, 092108 (21 pages), (2013).

[10] M. Lappa, Assessment of the role of axial vorticity in the formation of Particle Accumulation Structures in supercritical Marangoni and hybrid thermocapillary-rotation-driven flows, Phys. Fluids, 25(1), 012101, (2013). - M. Lappa, On the Existence and Multiplicity of One-dimensional Solid Particle Attractors in Timedependent Rayleigh-Bénard Convection, Chaos, 23(1), 013105, (2013).

[11] M. Lappa, On the variety of particle accumulation structures under the effect of gitters, J. Fluid Mech., 726, 160-195, (2013).

[12] S. Ravichandran, P. Perlekar and R. Govindarajan, Attracting fixed points for heavy particles in the vicinity of a vortex pair, Phys. Fluids 26, 013303, (2014) -

[13] M. Lappa, Stationary Solid Particle Attractors in Standing Waves, Phys. Fluids, 26(1), 013305 (12 pages), (2014)

[14] S.S. Tabakova and Z.D. Zapruanov, On the hydrodynamic interaction of two spheres oscillating in a viscous fluid. I. Axisymmetrical case. J. Appl. Math. Phys. (ZAMP), vol. 33, pp. 344-357, (1982). S.S. Tabakova and Z.D. Zapruanov, On the hydrodynamic interaction of two spheres oscillating in a viscous fluid. II. Three dimensional case. J. Appl. Math. Phys. (ZAMP), vol. 33, pp. 487-502 (1982).

[15] D. Langbein, Motion of Ensembles of Spherical Particles in a Fluid Due to g-jitter, Adv. Space Res., 11(7), 189-196, (1991).

[16] L. Petit and B. Noetinger, Shear induced structures in macroscopic dispersions, Rheol. Acta, 27(4), 437441, (1988).

[17] P. Evesque, "Sablier inversé", Pour la Science, 239, 94-96, (1997).

[18] M. Tirumkudulu, A. Tripathi, A. Acrivos, Particle segregation in monodisperse sheared suspensions, Phys. Fluids, 11(3), 507-509, (1999).

[19] R. Wunenburger, V. Carrier, and Y. Garrabos, Periodic order induced by horizontal vibrations in a twodimensional assembly of heavy beads in water, Phys. Fluids, 14, 2350, (2002).

[20] Y-J. Zhu and Y-Q. Ma, Orientational structures of a phase-separating system under oscillatory particles, J. Chem. Phys. 117, 10207, (2002).

[21] G. A. Voth, B. Bigger, M.R. Buckley, W. Losert, M.P. Brenner, H.A. Stone, and J.P. Gollub, Ordered clusters and dynamical states of particles in a vibrated fluid, Phys. Rev. Lett., 88, 234301 (2002).

[22] J.R. Thomson, J. Casademunt, F. Drolet and J. Vinals, Coarsening of solid-liquid mixtures in a random acceleration field, Phys Fluids, 9(5), 1336-1343, (1997).

[23] A.A. Ivanova and V.G. Kozlov, Sand-fluid interface under action of vibration. Fluid Dynamics, 37 (2), 277-293, (2002). A.A. Ivanova;V.G. Kozlov and A.F. Kuzaev, Vibrational lift force acting on a body in a fluid near a solid surface. Dokladi RAN, vol. 402 (4), pp. 1-4 (Translated: Doklady Physics, vol. 50 (6), pp. 311-314), (2005).

[24] V.G. Kozlov, A.A. Ivanova and P. Evesque, Block stratification of sedimenting granular matter in a vessel due to vertical vibration, Fluid Dyn. Mater. Process., 2(3), 203-210, (2006).

[25] S. Hassan, T. P. Lyubimova, D. V. Lyubimov, M. Kawaji, Effects of vibrations on particle motion near a wall: Existence of attraction force, Int. J. Multiphase Flow, 32 (9), 1037-1054, (2006).

[26] S. Hassan and M. Kawaji, The Effects of Vibrations on Particle Motion in a Viscous Fluid Cell, J. Appl. Mech., 75, 031012 [7 pages], (2008).

[27] M. Saadatmand, M. Kawaji, Effect of Viscosity on Vibration-Induced Motion of a Spherical Particle Suspended in a Fluid Cell, Microgravity Sci. Technol. (2010) 22:433-440, (2010).

[28] D. J. Pine, J. P. Gollub, J. F. Brady, and A. M. Leshansky, Chaos and threshold for irreversibility in sheared suspensions, Nature (London) 438, 997-1000, (2005).

[29] B. Metzger and J. E. Butler, Irreversibility and chaos: Role of long-range hydrodynamic interactions in sheared suspensions, Phys. Rev. E, 82, 051406, (2010).- B. Metzger, P. Pham and J. E. Butler, Irreversibility and chaos: Role of lubrication interactions in sheared suspensions, Phys. Rev. E, 87, 052304, (2013).

[30] B. Metzger and J. E. Butler, Clouds of particles in a periodic shear flow, Phys. Fluids., 24, 021703 (6 pages), (2012). L. Corté, P. M. Chaikin, J. P. Gollub and D. J. Pine, Random organization in periodically driven systems, Nature Physics, 4, 420-424 (2008) 
[31] H. A. Pacheco-Martinez, L. Liao, R. J. A. Hill, M.R. Swift, and R. M. Bowley, Spontaneous Orbiting of Two Spheres Levitated in a Vibrated Liquid, Phys. Rev. Lett. 110, 154501 (2013)

[32] R. Monti and R. Savino, Microgravity experiment acceleration tolerability on space orbiting laboratories, Journal of spacecraft and rockets, 33(5), 707-716 (1996). R. Savino and R. Monti, Convection induced by residual-g and g-jitters in diffusion experiments, Int. J. Heat Mass Transfer, 42(1), 111-126 (1999)

[33] R. Monti, R. Savino, M. Lappa, Microgravity sensitivity of typical fluid physics experiment, presented at the 17 th Microgravity Measurements Group Meeting, Cleveland, Ohio, 24-26 March 1998, published in the meeting proceedings in NASA CP-1998-208414, (23): 1-15 (ISSN: 0191-7811) (1998).

[34] R. Monti, R. Savino, M. Lappa, On the convective disturbances induced by g-jitter on the space station, Acta Astronautica, 48 (5-12), 603-615, (2001).

[35] M. Lappa and L. Carotenuto, Effect of convective disturbances induced by g-jitter on the periodic precipitation of lysozyme, Microgravity Science \& Technology, XIV/2, 41-56, (2003).

[36] S. Mazzoni, V. Shevtsova, A. Mialdun, D. Melnikov, Yu. Gaponenko, T. Lyubimova and Z. Saghir, Vibrating liquids in space, Europhysics News, Vol. 41 (6), pp. 14-16, (2010)

[37] R. Savino, M. Lappa, Assessment of the thermovibrational theory: application to g-jitter on the Spacestation, Journal of Spacecraft and Rockets, 40 (2), 201-210, (2003).

[38] V. Shevtsova, A. Mialdun, D. Melnikov, I. Ryzhkov, Y. Gaponenko, Z. Saghir, T. Lyubimova, J.C. Legros, The IVIDIL experiment onboard the ISS: Thermodiffusion in the presence of controlled vibrations, Comptes Rendus Mécanique, Vol. 339 (5), pp. 310-317, (2011) - V. Shevtsova, T. Lyubimova, Z. Saghir, D. Melnikov, Y. Gaponenko, V. Sechenyh, J.C. Legros and A. Mialdun, IVIDIL: on-board g-jitters and diffusion controlled phenomena, J. Phys.: Conf. Ser., Vol. 327, 012031, (2011)

[39] B. Maryshev, T. Lyubimova, and D. Lyubimov, (2013), Two-dimensional thermal convection in porous enclosure subjected to the horizontal seepage and gravity modulation, Phys. Fluids 25, 084105 (2013)

[40] M. Lappa, Secondary and oscillatory gravitational instabilities in canonical three-dimensional models of crystal growth from the melt, Part1: Rayleigh-Bènard systems, Comptes Rendus Mécanique, 335(5-6), 253260, (2007). - M. Lappa, Secondary and oscillatory gravitational instabilities in canonical three-dimensional models of crystal growth from the melt, Part2: Lateral heating and the Hadley circulation, Comptes Rendus Mécanique, 335(5-6), 261-268, (2007).

[41] M. Lappa, Some considerations about the symmetry and evolution of chaotic Rayleigh-Bénard convection: The flywheel mechanism and the "wind" of turbulence, Comptes Rendus Mécanique, 339, 563572, (2011). Lappa M., (2005), Thermal convection and related instabilities in models of crystal growth from the melt on earth and in microgravity: Past history and current status, Cryst. Res. Technol. (ISSN: 02321300), 40(6): 531-549.

[42] A. Babiano, J. H. E. Cartwright, O. Piro and A. Provenzale, Dynamics of a small neutrally buoyant sphere in a fluid and targeting in Hamiltonian systems, Phys. Rev. Lett., 84, 5764-5767, (2000).

[43] H.C. Kuhlmann, M. Lappa, D. Melnikov, R. Mukin, F.H. Muldoon, D. Pushkin, V.S. Shevtsova, and I. Ueno, The JEREMI-Project on thermocapillary convection in liquid bridges. Part A: Overview of Particle Accumulation Structures, Fluid Dyn. Mater. Process., 10 (1), 1-36, (2014).

[44] M. Lappa, Oscillatory convective structures and solutal jets originated from discrete distributions of droplets in organic alloys with a miscibility gap, Physics of Fluids, 18 (4), 042105 (14 pages), (2006). M. Lappa, Growth and Mutual Interference of Protein Seeds under reduced gravity conditions, Physics of Fluids, 15 (4), 1046-1057, (2003).

[45] M. Lappa and R. Savino, Parallel solution of the three-dimensional Marangoni flow instabilities in liquid bridges, Int. J. Num. Meth. Fluids, 31(6), 911-925, (1999).

[46] K.A. Atkinson, An Introduction to Numerical Analysis (2nd ed.), (John Wiley \& Sons, New York, 1989).

[47] D. Beysens, R. Wunenburger, C. Chabot, and Y. Garrabos, Effect of oscillatory accelerations on twophase fluids, Microgravity Sci. Technol., 11, 113-118, (1998).

[48] V. M. B. Nunes, M. J. V. Lourenço, F. J. V. Santos and C. A. Nieto de Castro, Viscosity of Molten Sodium Nitrate, International Journal of Thermophysics, 27(6), 1638-1649, (2006).

[49] A. Mialdun, I. Ryzhkov, D.E. Melnikov, and V. Shevtsova, Experimental Evidence of Thermal Vibrational Convection in a Nonuniformly Heated Fluid in a Reduced Gravity Environment, Phys. Rev. Lett., 101, 084501, (2008).

[50] M.K. Bennett, Affine and Projective Geometry. (Wiley, New York, 1995). 